This manuscript has been accepted for publication in Computational Materials Science (DOI: 10.1016/j.commatsci.2014.11.057). The manuscript will undergo copyediting, typesetting, and review of the resulting proof before it is published in its final form. Please note that during the production process errors may be discovered which could affect the content, and all disclaimers that apply to the journal apply to this manuscript. A definitive version will be subsequently published. 


\title{
A quantitative phase-field model for two-phase elastically inhomogeneous systems
}

\author{
A. Durga*, P. Wollants, N. Moelans \\ Department of Materials Engineering, Faculty of Engineering, \\ KU Leuven, Kasteelpark Arenberg 44 Bus 2450, BE-3001 Leuven, Belgium
}

\begin{abstract}
Solid-state phase transformations are influenced by strains that are generated internally or applied externally. The stress state, composition, and microstructure evolution, which together determine the properties of solid materials can be studied using phase-field models coupled with micro-elasticity theory in the small strain limit. This coupling has been implemented using various schemes in literature. In a previous article (Durga et al., 2013 [1]), the authors evaluated three main existing schemes for a two-phase system and concluded that these schemes are not quantitative for inhomogeneous anisotropic elastic properties of the two phases. The stress states predicted by these models deviate from the expected values due to the generation of extra interfacial energy, which is an artefact of the models resulting from interfacial conditions different from local mechanical equilibrium conditions. In this work, we propose a new scheme with interfacial conditions consistent with those of the analytical results applicable to a general system where shear strains may be present. Using analytical solutions for composition and stress evolution, we validate this model for 2D and 3D systems with planar interface in the presence of misfit between phases and applied strains, and a 2D system with an elliptical second-phase particle. This extended scheme can now be applied to simulate quantitatively the microstructural evolution with coupled chemical and mechanical behaviour in any $2 \mathrm{D}$ or $3 \mathrm{D}$ two-phase system subject to internal or external strains irrespective of interface curva-
\end{abstract}

\footnotetext{
${ }^{*}$ Corresponding author; tel: +32-16-373734

Email addresses: durga87@gmail.com (A. Durga), patrick.wollants@mtm.kuleuven.be (P. Wollants), nele.moelans@mtm.kuleuven.be (N. Moelans)
} 
ture.

Keywords: Phase-field model, Coherent interfaces, Microstructure evolution, Phase transformation, Anisotropic elasticity, Interface energy

\section{Introduction}

Phase-field models are used to solve moving boundary problems such as phase transformations in solid and liquid states and transport phenomena involving fluid flow or mass diffusion, which involve elastic, magnetic, electrochemical and other physical effects. The main advantage of this method is the use of a diffuse interface between different domains. This is represented by one or more phase-field variables which vary continuously across the interface. The evolution of the system is then driven by the minimisation of total free energy, which is a function of the phase-field variables in space and time. In this article, a quantitative phase-field model considering the effects of chemical diffusion and linear elasticity on microstructure evolution in solid-state materials is presented.

Phase-field models coupled with microelasticity theory have been extensively applied to study various phenomena such as martensite transformations [2], grain growth, and texture evolution [3]. Several schemes are available in literature that combine microelasticity theory with phase-field models. Steinbach-Apel's scheme (SAS) [4], Voigt-Taylor's scheme (VTS) [5, 6], and Khachaturyan's scheme (KHS) [7] are the three main existing schemes. They differ in the way the elastic strain, stress, and elastic constants are defined in the diffuse interface, which in turn causes a difference in the elastic contribution to the driving force for the system evolution. There have been only limited $[4,5,8]$ comparative studies of these schemes with analytical solutions, especially for complex morphologies of inhomogeneous systems and the coupling with chemical equilibrium.

In 'thin interface' phase-field models [9], the interface width is generally taken abnormally large compared to the actual physical interface width in materials. In order to provide reliable results, we need to use quantitative models, i.e. those that do not depend on the value of the diffuse interface width used in the phase-field model. The results have to be independent of the value of the interface width as long as the microstructural features of interest are larger than the interface width. Such quantitative phasefield models are available for studying phase transformations and diffusion 
in multiphase systems [10], solidification [11] and grain growth [12]. These models ensure that the bulk energy of the system is decoupled from the interfacial energy that arises due to the diffuse interface. Coupling phasefield models with elasticity, however, presents a complication due to the presence of many inter-related tensorial quantities such as the elastic strain, the elastic stress, stiffness, and the displacement fields. Therefore, a simple analogy with the other quantitative models does not exist. Examples of quantitative phase-field models with tensorial fields include that of Nicoli et al. [13], which uses mobility tensor for a two-phase system with different interpolations for different components of the tensor, and Yeon et al. [8], wherein a quantitative elastic phase-field model for binary coherent twophase systems with stiffnesses of cubic symmetry is presented.

In a previous work [1], the authors studied a two-phase inhomogeneous system separated by a planar interface and showed that the interpolation of elastic properties in phase-field models following the schemes of KHS, SAS, and VTS are not quantitative, i.e., the bulk properties depend on the diffuse interface width used in the model and excess interfacial energy is created due to the elastic energy formulation. The three schemes are quantitative only under special circumstances: KHS when the elastic strains are equal in the two phases, SAS when the stresses are equal in the two phases and VTS when the total strains are equal in the two phases. We then introduced a new quantitative scheme which uses interfacial conditions that are consistent with the analytical results. We validated this scheme for a 2-D two-phase system with a planar interface in the absence of shear strains. However, in order to be applicable to a general two-phase system with arbitrary interface curvatures and shear strains in $2-\mathrm{D}$ and $3-\mathrm{D}$, the model needs to be further developed and validated.

The aim of this work is to extend and validate the new scheme for $2 \mathrm{D}$ and $3 \mathrm{D}$ two-phase systems with shear strains, arbitrary interface curvature, and no restriction on the nature of the elastic constants. The model is validated for the following cases of two-phase systems using corresponding analytical solutions: (i) planar interface with shear strains and applied strains in 2D and 3D (Johnson's analytical model [14]) and (ii) elliptical precipitate in a large matrix in 2D (Jin et al.'s analytical solution [15]).

The rest of the paper is organised as follows. First, we discuss analytical solutions available for 2D and 3D systems in Section 2. Then, the phase-field model used in this study is given in Section 3. The coupling of elastic energy with the phase-field model using the new quantitative scheme is formulated 
in Section 4. Section 5 discusses the simulation results for the different cases and the main conclusions are drawn in Section 6.

\section{Analytical solutions}

Starting with a brief introduction to microelasticity theory, we present analytical solutions available in the literature for 3 cases: Johnson's description [14] for 2D and 3D systems with planar interface, and Jin et al.'s solution [15] for a 2D system with an elliptical second-phase precipitate. These will be compared with results from the phase-field simulations in order to validate the model.

\subsection{Microelasticity theory}

We consider a coherent two-phase system. No assumptions are made about the nature of the elastic moduli. Undeformed $\alpha$ phase is taken as the reference state for the calculation of eigenstrain. The stresses and strains are defined in the system according to Khachaturyan [7]. The elastic stress in the small-strain regime, where linear elasticity theory holds, is given by:

$$
\sigma_{i j}=C_{i j k l} \epsilon_{k l}^{e l}
$$

where $C_{i j k l}$ is the stiffness tensor and $\epsilon_{k l}^{e l}$ the elastic strain. Einstein summation notation is used for all equations involving tensors in this article.

Elastic strain is defined as:

$$
\epsilon_{k l}^{e l}=\epsilon_{k l}-\epsilon_{k l}^{*}=\overline{\epsilon_{k l}}+\delta \epsilon_{k l}-\epsilon_{k l}^{*},
$$

where $\epsilon_{k l}=\overline{\epsilon_{k l}}+\delta \epsilon_{k l}$ is the total strain. $\epsilon_{k l}^{*}$ is the eigenstrain given by the relative difference in the lattice parameters of the two phases. Taking underformed $\alpha$ as the reference state, the eigenstrains are then zero in the $\alpha$ phase and typically non-zero in the $\beta$ phase. $\overline{\epsilon_{k l}}$ is the homogeneous or applied strain defined such that

$$
\int_{V} \delta \epsilon_{k l} \mathrm{~d}^{3} \mathrm{r}=0
$$

The heterogeneous strain $\delta \epsilon_{k l}$ is related to the local displacement fields $u_{i}(\vec{r})$ as

$$
\delta \epsilon_{k l}=\frac{1}{2}\left[\frac{\partial u_{k}(\vec{r})}{\partial r_{l}}+\frac{\partial u_{l}(\vec{r})}{\partial r_{k}}\right]
$$


The elastic energy density is then given by:

$$
f^{e l}=\frac{1}{2} \epsilon_{i j}^{e l} C_{i j k l} \epsilon_{k l}^{e l} .
$$

At mechanical equilibrium, $\frac{\partial \sigma_{i j}}{\partial r_{j}}=0$.

\subsection{Johnson's model}

Johnson's description gives the conditions for interfacial mechanical and chemical equilibrium. In Sections 2.2.1 and 2.2.2, the mechanical equilibrium is given for $2 \mathrm{D}$ and $3 \mathrm{D}$ systems with planar interfaces respectively. In Section 2.2.3, the equilibrium compositions in the presence of strains is given, which can be calculated on knowing the stress state of the system.

\subsubsection{D system with misfit between phases and applied strains}

Considering a 2D rectangular two-phase system as shown in Fig. 1(a), the equilibrium interfacial relations for the stresses and strains are as follows. There is continuity of displacements and tractions at the interface:

$$
\begin{aligned}
u_{i}^{\alpha} & =u_{i}^{\beta}, \\
\sigma_{i j}^{\alpha} n_{j}^{\alpha}+\sigma_{i j}^{\beta} n_{j}^{\beta} & =0,
\end{aligned}
$$

where $n_{j}$ are the components of the outward pointing unit normals to the respective phases at the interface. From the geometry, $n_{1}^{\alpha}=-n_{1}^{\beta}$ and $n_{2}^{\alpha}=$ $n_{2}^{\beta}=0$. For $i=1,(7)$ gives $\sigma_{11}^{\alpha}=\sigma_{11}^{\beta}$, and for $i=2, \sigma_{12}^{\alpha}=\sigma_{12}^{\beta}$. Expanding these two equalities, we get

$$
\begin{aligned}
& C_{1111}^{\alpha}\left(\bar{\epsilon}_{11}+\delta \epsilon_{11}^{\alpha}-\epsilon_{11}^{*, \alpha}\right)+C_{1122}^{\alpha}\left(\bar{\epsilon}_{22}+\delta \epsilon_{22}^{\alpha}-\epsilon_{22}^{*, \alpha}\right)+2 C_{1112}^{\alpha}\left(\bar{\epsilon}_{12}+\delta \epsilon_{12}^{\alpha}-\epsilon_{12}^{*, \alpha}\right) \\
= & C_{1111}^{\beta}\left(\bar{\epsilon}_{11}+\delta \epsilon_{11}^{\beta}-\epsilon_{11}^{*, \beta}\right)+C_{1122}^{\beta}\left(\bar{\epsilon}_{22}+\delta \epsilon_{22}^{\beta}-\epsilon_{22}^{*, \beta}\right)+2 C_{1112}^{\beta}\left(\bar{\epsilon}_{12}+\delta \epsilon_{12}^{\beta}-\epsilon_{12}^{*, \beta}\right), \\
& C_{1112}^{\alpha}\left(\bar{\epsilon}_{11}+\delta \epsilon_{11}^{\alpha}-\epsilon_{11}^{*, \alpha}\right)+C_{2212}^{\alpha}\left(\bar{\epsilon}_{22}+\delta \epsilon_{22}^{\alpha}-\epsilon_{22}^{*, \alpha}\right)+2 C_{1212}^{\alpha}\left(\bar{\epsilon}_{12}+\delta \epsilon_{12}^{\alpha}-\epsilon_{12}^{*, \alpha}\right) \\
= & C_{1112}^{\beta}\left(\bar{\epsilon}_{11}+\delta \epsilon_{11}^{\beta}-\epsilon_{11}^{*, \beta}\right)+C_{2212}^{\beta}\left(\bar{\epsilon}_{22}+\delta \epsilon_{22}^{\beta}-\epsilon_{22}^{*, \beta}\right)+2 C_{1212}^{\beta}\left(\bar{\epsilon}_{12}+\delta \epsilon_{12}^{\beta}-\epsilon_{12}^{*, \beta}\right) .
\end{aligned}
$$

With the no-slip condition in this geometry, the displacements do no vary in the ' 2 ' direction. Therefore, $\delta \epsilon_{22}^{\alpha}=\frac{\partial u_{2}^{\alpha}}{\partial r_{2}}=0=\frac{\partial u_{2}^{\beta}}{\partial r_{2}}=\delta \epsilon_{22}^{\beta}$.

From (3), when the areas of $\alpha$ and $\beta$ phase are equal,

$$
\begin{aligned}
& \delta \epsilon_{11}^{\alpha}=-\delta \epsilon_{11}^{\beta}, \\
& \delta \epsilon_{12}^{\alpha}=-\delta \epsilon_{12}^{\beta} .
\end{aligned}
$$




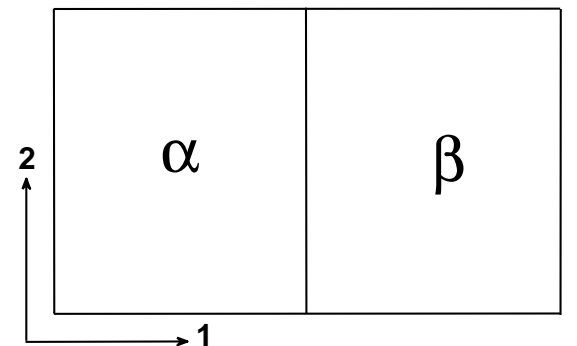

(a)

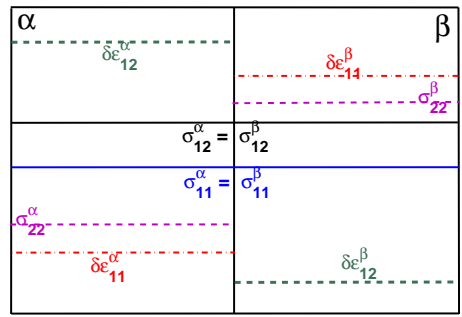

(b)

Figure 1: Illustration of a rectangular 2-phase 2-D system: (a) Configuration. (b) Strain and stress profiles as calculated from Johnson's model (only non-zero quantities displayed).

From (8), (9), (10) and (11), we can solve for $\delta \epsilon_{11}^{\alpha}$ and $\delta \epsilon_{12}^{\alpha}$. From the global mechanical equilibrium condition, $\frac{\partial \sigma_{i j}}{\partial r_{j}}=0$, all the strain components and thereby, the stress components, are constant within their respective phases. The non-zero heterogeneous strain and elastic stress components in the two phases are illustrated in Fig. 1(b).

\subsubsection{D system with misfit between phases and applied strains}

For a 3D system with equal volumes of $\alpha$ and $\beta$ phases separated by a planar interface as shown in Fig. 2(a), we can follow the same principles as in the $2 \mathrm{D}$ system.

Here, the relations between the components of the interface normals are: $n_{1}^{\alpha}=-n_{1}^{\beta}, n_{2}^{\alpha}=n_{2}^{\beta}=0$ and $n_{3}^{\alpha}=n_{3}^{\beta}=0$. We then get the following relations for the different stress components on applying the force balance equation (7) at equilibrium:

$$
\begin{aligned}
& \sigma_{11}^{\alpha}=\sigma_{11}^{\beta}, \\
& \sigma_{12}^{\alpha}=\sigma_{12}^{\beta}, \\
& \sigma_{13}^{\alpha}=\sigma_{13}^{\beta} .
\end{aligned}
$$

It follows from the no-slip condition for this geometry that all the displacements are constant along ' 2 ' and ' 3 ' directions. Therefore, only the partial derivatives of the displacements with respect to $r_{1}$ are non-zero. The 


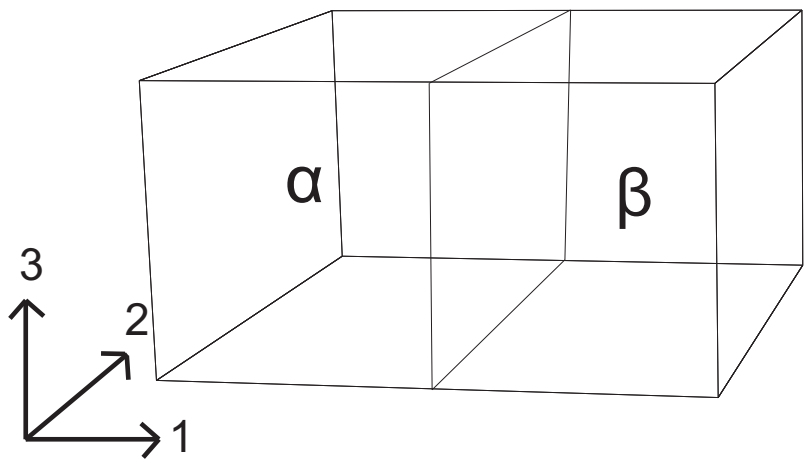

(a)

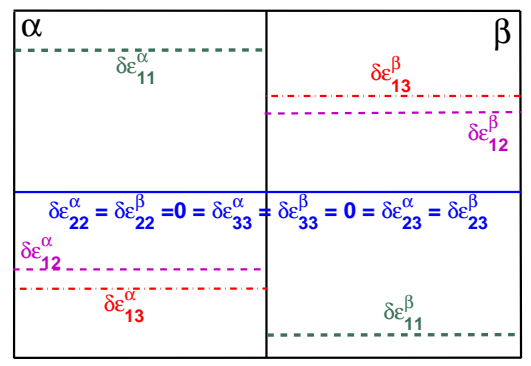

(b)

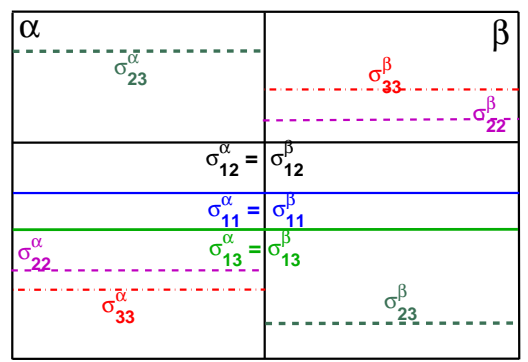

(c)

Figure 2: Illustration of a cuboidal 2-phase 3-D system: (a) Configuration. (b) Stress and (c) strain profiles as calculated from Johnson's model.

following strain components are then equal to zero:

$$
\begin{array}{r}
\delta \epsilon_{22}^{\alpha}=\frac{\partial u_{2}^{\alpha}}{\partial r_{2}}=0=\frac{\partial u_{2}^{\beta}}{\partial r_{2}}=\delta \epsilon_{22}^{\beta}, \\
\delta \epsilon_{23}^{\alpha}=\frac{1}{2}\left[\frac{\partial u_{2}^{\alpha}}{\partial r_{3}}+\frac{\partial u_{3}^{\alpha}}{\partial r_{2}}\right]=0=\frac{1}{2}\left[\frac{\partial u_{2}^{\beta}}{\partial r_{3}}+\frac{\partial u_{3}^{\beta}}{\partial r_{2}}\right]=\delta \epsilon_{23}^{\beta}, \\
\delta \epsilon_{33}^{\alpha}=\frac{\partial u_{3}^{\alpha}}{\partial r_{3}}=0=\frac{\partial u_{3}^{\beta}}{\partial r_{3}}=\delta \epsilon_{33}^{\beta} .
\end{array}
$$


When the areas of $\alpha$ and $\beta$ phase are equal, (3) gives

$$
\begin{aligned}
& \delta \epsilon_{11}^{\alpha}=-\delta \epsilon_{11}^{\beta}, \\
& \delta \epsilon_{12}^{\alpha}=-\delta \epsilon_{12}^{\beta}, \\
& \delta \epsilon_{13}^{\alpha}=-\delta \epsilon_{13}^{\beta} .
\end{aligned}
$$

These non-zero heterogeneous strain components can be calculated by expanding (12) - (14) and using (18) - (20). As a result of the global mechanical equilibrium condition, $\frac{\partial \sigma_{i j}}{\partial r_{j}}=0$, all the strain components and, thereby, the stress components are constant within their respective phases. The strain and stress components are illustrated in Figs. 2(b) and 2(c) respectively.

\subsubsection{Change in equilibrium composition}

In the presence of strains, the chemical equilibrium composition changes [16] since the total free energies of the phases also change. Knowing the stress state in the different phases, the new equilibrium compositions at the interface, $c_{\alpha}^{e}$ and $c_{\beta}^{e}$, are given by:

$$
\begin{aligned}
c_{\alpha}^{e} & =c_{\alpha, 0}+\frac{\left(\sigma_{k l}^{\beta} \epsilon_{k l}^{e l, \beta}-\sigma_{k l}^{\alpha} \epsilon_{k l}^{e l, \alpha}\right) / 2+\left(\epsilon_{k l}^{e l, \alpha}-\epsilon_{k l}^{\beta}\right) \sigma_{k l}^{\alpha}+\kappa_{c} \sigma_{i n t}}{A^{\alpha}\left(c_{\beta, 0}-c_{\alpha, 0}\right)}, \\
c_{\beta}^{e} & =c_{\beta, 0}+\frac{\left(\sigma_{k l}^{\beta} \epsilon_{k l}^{e l, \beta}-\sigma_{k l}^{\alpha} \epsilon_{k l}^{e l, \alpha}\right) / 2+\left(\epsilon_{k l}^{e l, \alpha}-\epsilon_{k l}^{\beta}\right) \sigma_{k l}^{\alpha}+\kappa_{c} \sigma_{i n t}}{A^{\beta}\left(c_{\beta, 0}-c_{\alpha, 0}\right)}
\end{aligned}
$$

where $A^{\alpha}$ and $A^{\beta}$ are the second derivatives of the chemical energy densities, $c_{\alpha, 0}$ and $c_{\beta, 0}$ are the equilibrium compositions of the two phases in the absence of strains, $\kappa_{c}$ is the mean interface curvature $(=1 /$ mean radius of the $\beta$ phase particle; $=0$ if the interface is planar), and $\sigma_{\text {int }}$ the interfacial energy.

\subsection{Analytical solution for an elliptical inhomogeneity in plane elasticity}

Jin et al. [15] have derived the elastic field generated by an elliptical inhomogeneity in a 2D infinite matrix subjected to an externally applied stress. In this work, we use their method and solution for a slightly modified $2 \mathrm{D}$ system. We assume that there is no externally applied stress but the elliptical inhomogeneous precipitate $(\beta)$ has a dilatational eigenstrain $\epsilon_{k l}^{*, \beta}$ due to its different lattice parameter compared to the infinite matrix $(\alpha)$ phase. Both phases are assumed to have isotropic but different stiffnesses. The solutions are expressed by introducing an 'equivalent homogeneous inclusion', 
i.e., a precipitate with stiffness equal to that of the matrix, in place of the original inhomogeneous precipitate. This equivalent homogeneous inclusion then has pseudo-eigenstrains $\left(\epsilon_{k l}^{* p}\right)$ such that the same forces are exerted on the matrix as in the case of the original inhomogeneous precipitate and the precipitate undergoes the same net deformation, given by $\delta \epsilon_{k l}^{\beta}$. Thus, the equilibrium stresses in the precipitate are the same in the two cases:

$$
\sigma_{i j}^{\beta}=2 \mu^{\alpha}\left(\delta \epsilon_{k l}^{\beta}-\epsilon_{k l}^{* p}\right)+\lambda^{\alpha}\left(\delta \epsilon_{k k}^{\beta}-\epsilon_{k k}^{* p}\right)=2 \mu^{\beta}\left(\delta \epsilon_{k l}^{\beta}-\epsilon_{k l}^{*, \beta}\right)+\lambda^{\beta}\left(\delta \epsilon_{k k}^{\beta}-\epsilon_{k k}^{*, \beta}\right),
$$

where $\mu^{\alpha}$ and $\mu^{\beta}$ are the shear moduli, and $\lambda^{\alpha}$ and $\lambda^{\beta}$ are the Lamé's first parameters of the two phases. The pseudo-eigenstrains $\epsilon_{k l}^{* p}$ are solved by expanding this equation for all stress components and eliminating the heterogeneous strains in the precipitate which are given by equations (13) and (14) in [15]. This solution is the same as that obtained from Jaswon and Bhargava [17] who solve this problem using a complex variable formalism, thereby validating the method given by Jin et al. [15] for this particular problem also. It is then possible to calculate the elastic strains and stresses, all of which are constant inside the precipitate as shown by Eshelby [18].

The stresses in the matrix are not constant and are given by equations (23)-(26) in [15] using the pseudo-eigenstrains. The stress profiles along the semi-major axis of the ellipse are illustrated in Fig. 3(b). We can see that there is continuity of the $\sigma_{11}$ and $\sigma_{12}$ (= zero) components of the stress, and a discontinuity of $\sigma_{22}$ component at the interface between the precipitate and the matrix, as expected at mechanical equilibrium. All stress components tend to zero in the matrix far away from the ellipse.

On knowing the stress state of the system, we can calculate the composition change at the interface from (21) and (22) using an 'equivalent circle radius' for the ellipse.

\section{Phase-field model}

We consider binary (A-B) two-phase $(\alpha-\beta)$ systems in $2 \mathrm{D}$ and $3 \mathrm{D}$ with arbitrary interface curvatures. In order to study both microstructure and composition evolution in the presence of strains, we consider one conserved variable (composition $c$ given as molar fraction of B) and one non-conserved phase-field variable $(\phi)$. $\phi$ takes the value 1 in the $\alpha$ phase, 0 in the $\beta$ phase and a range of values between these two at the interface. The temperature 


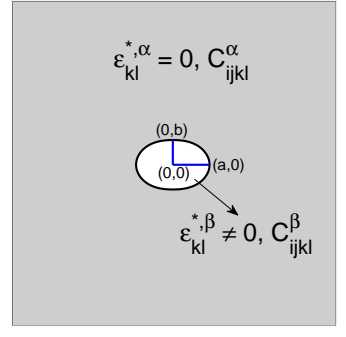

(a)

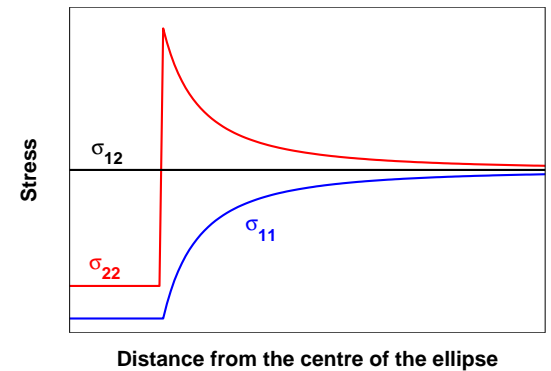

(b)

Figure 3: Illustration of a 2-D system with an elliptical precipitate in an infinite matrix: (a) Configuration. (b) Stress profiles as calculated using Jin et al.'s [15] solution: Continuity of $\sigma_{11}$ and $\sigma_{12}$ and discontinuity of $\sigma_{22}$ at the interface.

is assumed to be constant in the system and thermal effects are not considered. There are then three contributions to the total free energy functional: interfacial $\left(f^{i n t}\right)$, chemical $\left(f^{c h}\right)$ and elastic $\left(f^{e l}\right)$ energy densities. The total energy is given as:

$$
F=\int_{V}\left(f^{i n t}+f^{c h}+f^{e l}\right) \mathrm{d} V .
$$

The chemical and interfacial energies are given by Kim et al.'s [11] model:

$$
\begin{array}{r}
f^{i n t}=\frac{\kappa}{2}(\vec{\nabla} \phi)^{2}+W g(\phi), \\
f^{c h}=p(\phi) f_{\alpha}^{c h}+[1-p(\phi)] f_{\beta}^{c h},
\end{array}
$$

using the interpolation function $p(\phi)=\phi^{3}\left(6 \phi^{2}-15 \phi+10\right) . \kappa$ is the gradient energy coefficient, $g(\phi)=\phi^{2}(1-\phi)^{2}$, and $\mathrm{W}$ the depth of this double-well function. $\kappa$ and $W$ determine the interfacial energy and the width of the diffuse interface. In this study, the Gibbs energy densities of the two phases are assumed to be parabolic functions of the composition with minima at $c_{\alpha, 0}$ and $c_{\beta, 0}$ respectively:

$$
\begin{array}{r}
f_{\alpha}^{c h}=\frac{A^{\alpha}}{2}\left(c_{\alpha}-c_{\alpha, 0}\right)^{2}, \\
f_{\beta}^{c h}=\frac{A^{\beta}}{2}\left(c_{\beta}-c_{\beta, 0}\right)^{2}, \\
c=p(\phi) c_{\alpha}+[1-p(\phi)] c_{\beta} .
\end{array}
$$


At every point in the system, the diffusion potential is assumed to be equal in the two phases: $\mu_{\alpha}=\mu_{\beta}=\frac{\partial f^{c h}}{\partial c}=\mu$, which ensures local chemical equilibrium. Using this relation and (29), the phase compositions $c_{\alpha}$ and $c_{\beta}$ are computed [11].

The elastic energy is given by (5). In order to couple microelasticity theory with the phase-field method, the stress, strain and elastic energy density must be interpolated and defined at each point of the diffuse interface. The elastic energy density is interpolated as:

$$
f^{e l}=p(\phi) f_{\alpha}^{e l}+[1-p(\phi)] f_{\beta}^{e l},
$$

where $f_{\alpha}^{e l}=\frac{1}{2} \epsilon_{i j}^{e l, \alpha} C_{i j k l}^{\alpha} \epsilon_{k l}^{e l, \alpha}$ and $f_{\beta}^{e l}=\frac{1}{2} \epsilon_{i j}^{e l, \beta} C_{i j k l}^{\beta} \epsilon_{k l}^{e l, \beta}$ are the elastic energies of the two phases. $C_{i j k l}^{\alpha}$ and $C_{i j k l}^{\beta}$ are the stiffness tensors of the two phases and $\epsilon_{k l}^{e l, \alpha}$ and $\epsilon_{k l}^{e l, \beta}$, the elastic strains in the two phases. The stresses and strains are interpolated complying with the elastic energy interpolation using different schemes as given in Section 4. All elastic properties are assumed to be functions of the different phases, i.e., the phase field variable only, and independent of composition. However, the composition is indirectly coupled to the elastic strain through $\phi$ and the effect of the strains on the composition are captured through this indirect coupling.

For each scheme, the displacement field at mechanical equilibrium is first solved using $\frac{\partial \sigma_{i j}}{\partial r_{j}}=0$ at every time step since the chemical diffusion process is much slower than elastic displacements. Then, the elastic contribution to the driving force $\frac{\partial f^{e l}}{\partial \phi}$ is computed.

The temporal evolution of the composition and phase-field variables are given by:

$$
\begin{array}{r}
\frac{\partial c}{\partial t}=\vec{\nabla} \cdot\left(M \vec{\nabla} \frac{\delta F}{\delta c}\right)=\vec{\nabla} \cdot(M \vec{\nabla} \mu), \\
\frac{\partial \phi}{\partial t}=-L \frac{\delta F}{\delta \phi}=-L\left(\frac{\partial f^{i n t}}{\partial \phi}+\frac{\partial f^{c h}}{\partial \phi}+\frac{\partial f^{e l}}{\partial \phi}\right),
\end{array}
$$

where $\mathrm{M}$ is the diffusion mobility and $\mathrm{L}$ a kinetic parameter related to the interface mobility.

\section{Interpolation schemes for elastic energy formulation}

Several schemes exist in literature for interpolating the elastic properties. Two common assumptions are equal stress at the interface or equal 
total strain at the interface. In this section, the Steinbach-Apel's scheme (SAS) [4] and the Voigt-Taylor's scheme (VTS) [5, 6], which follow these two assumptions respectively, are described. Following a discussion of their shortcomings, an extended version (also considering shear strains) of the new scheme proposed by the authors in [1] is presented.

\subsection{Steinbach-Apel's scheme (SAS)}

In SAS, the elastic stress is assumed to be equal in the two phases (ReussSachs condition):

$$
\begin{aligned}
\sigma_{i j} & =\sigma_{i j}^{\alpha}=\sigma_{i j}^{\beta}, \\
\Longrightarrow \epsilon_{k l}^{e l} C_{i j k l}=\epsilon_{k l}^{e l, \alpha} C_{i j k l}^{\alpha} & =\epsilon_{k l}^{e l, \beta} C_{i j k l}^{\beta} .
\end{aligned}
$$

The elastic strain and eigenstrain are interpolated as:

$$
\begin{gathered}
\epsilon_{k l}^{e l}=p(\phi) \epsilon_{k l}^{e l, \alpha}+[1-p(\phi)] \epsilon_{k l}^{e l, \beta}, \\
\epsilon_{k l}^{*}=p(\phi) \epsilon_{k l}^{*, \alpha}+[1-p(\phi)] \epsilon_{k l}^{*, \beta} .
\end{gathered}
$$

Therefore,

$$
C_{i j k l}=\left[p(\phi) S_{i j k l}^{\alpha}+\{1-p(\phi)\} S_{i j k l}^{\beta}\right]^{-1},
$$

where $S_{i j k l}^{\alpha}=\left[C_{i j k l}^{\alpha}\right]^{-1}$ and $S_{i j k l}^{\beta}=\left[C_{i j k l}^{\beta}\right]^{-1}$ are the compliances of the two phases. The mechanical equilibrium equation, $\frac{\partial \sigma_{i j}}{\partial r_{j}}=0$, is then solved using the above relations in order to obtain the displacement fields, strains and stresses.

\subsection{Voigt-Taylor's scheme (VTS)}

In VTS, the total strain $\left(=\overline{\epsilon_{k l}}+\delta \epsilon_{k l}\right)$ is assumed to be equal in the two phases: $\epsilon_{k l}=\epsilon_{k l}^{\alpha}=\epsilon_{k l}^{\beta}$. The elastic strains of the two phases are then given by:

$$
\begin{aligned}
& \epsilon_{k l}^{e l, \alpha}=\epsilon_{k l}-\epsilon_{k l}^{*, \alpha}, \\
& \epsilon_{k l}^{e l, \beta}=\epsilon_{k l}-\epsilon_{k l}^{*, \beta} .
\end{aligned}
$$

The stress is interpolated as:

$$
\sigma_{k l}=p(\phi) \sigma_{k l}^{\alpha}+[1-p(\phi)] \sigma_{k l}^{\beta} .
$$

The mechanical equilibrium equation is solved using these assumptions in order to obtain the displacement fields, strains and stresses. 


\subsection{Shortcomings of $S A S$ and VTS}

In the previous article [1] by the authors, it was shown that both SAS and VTS give rise to excess interfacial energy and, therefore, the results depend on the interface width used in the phase-field model. This is because the conditions of equal stress or equal total strain at the interface are not true for all stress or strain components at equilibrium as shown in Section 2.

We simulated a $2 \mathrm{D} \mathrm{Cu}_{6} \mathrm{Sn}_{5}$-Bct-Sn system in the same configuration as Fig. 1(a). The stresses and strains are constant within each phase as calculated analytically in Section 2.2.1. Therefore, in the absence of excess energy due to the elastic energy formulation, a linear plot of elastic energy versus the interpolation function is expected. However, both SAS and VTS give rise to excess energy as shown in Fig. 4. Only the new scheme gives a linear relation. Moreover, the stress and strain values calculated using SAS and VTS do not always match the analytical values and vary with the interface width.

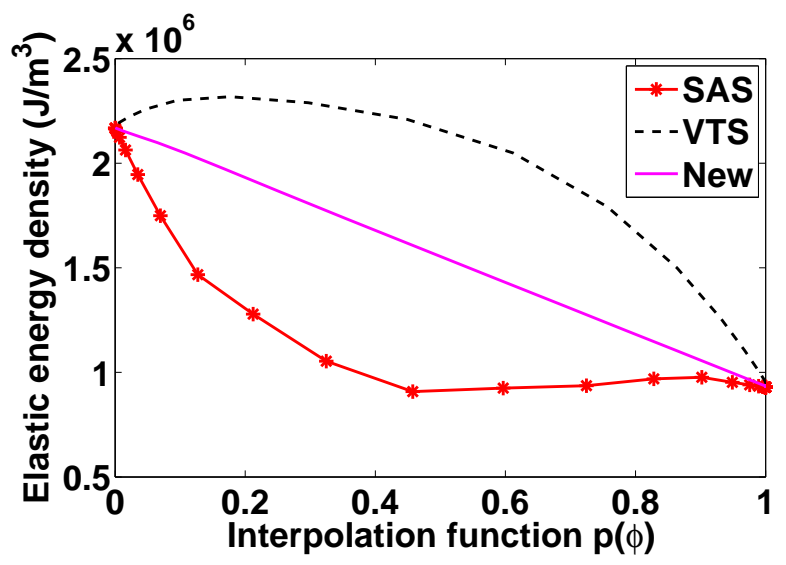

Figure 4: Elastic energy density as a function of $\mathrm{p}(\phi)$ obtained for different interpolation schemes for a simulation of a $2 \mathrm{D} \mathrm{Cu}_{6} \mathrm{Sn}_{5}$-Bct-Sn system with a planar interface: A linear dependence is expected in the absence of excess interfacial energy, i.e., in a quantitative model. Therefore, SAS and VTS are not quantitative, whereas the new scheme introduced in [1] is quantitative.

\subsection{New quantitative scheme}

Quantitative phase-field models have been constructed in the past using a description of properties at the diffuse interface that is consistent with the equilibrium interfacial conditions. For chemical energy, this is satisfied by 
forcing the diffusion potential (= first derivative of the chemical energy with respect to composition) to be equal in the two phases at the interface as proposed by Kim et al. [11] (see Section 3). However, for elastic energy, there is no such simple condition that can be implemented.

As described in Section 2, due to the tensorial nature of stresses and strains, only the $\sigma_{11}, \sigma_{12}$, and $\sigma_{13}$ stress components that are normal to the interface are equal in the two phases. Among the strain components, only $\delta \epsilon_{22}, \delta \epsilon_{23}$, and $\delta \epsilon_{33}$ are equal in the two phases at the interface. In the new scheme, in accordance with these equilibrium interfacial conditions, the stress components that are equal are calculated using SAS and the strain components that are equal are calculated using VTS. The other stress and strain components are derived from these known quantities. Therefore, in this scheme, we explicitly force the known interfacial conditions by using the stress and strain solutions obtained from SAS and VTS respectively.

One shortcoming of the scheme presented in our previous article is that shear stresses and strains were not considered since the $C_{1112}$ and $C_{2212}$ components of the stiffness tensor were assumed to be zero. For materials with monoclinic and triclinic crystal structures, even when there are no shear terms in the eigenstrain, shear stresses are generated due to non-zero offdiagonal terms in the stiffness tensor. For instance, for a phase with triclinic crystal structure, all components in the stiffness tensor are non-zero.

Also, in the case of arbitrary interface curvature between the two phases, shear strains are invariably generated. Therefore, shear strains also need to be considered in order for the new scheme to be applicable to materials with all types of morphologies and elastic constants. In this section, the new scheme is now extended and formulated for systems with shear strains and arbitrary interface curvatures.

Recently, Mosler et al. [19] have proposed a phase-field model for systems with large strains using a formulation of the energy that takes into account the continuity of displacements (6) and the continuity of tractions (7). However, the model has not been tested for different interface widths and the effect of strains on the chemical behaviour is not discussed.

\subsubsection{Model formulation for planar interfaces}

In SAS, it is assumed that $\sigma_{i j}^{\alpha}=\sigma_{i j}^{\beta}$ for all stress components. However, this is true only for $\sigma_{11}, \sigma_{12}$, and $\sigma_{13}$ at equilibrium as shown in Section 2 . In VTS, $\delta \epsilon_{i j}^{\alpha}\left(+\bar{\epsilon}_{i j}\right)=\delta \epsilon_{i j}^{\beta}\left(+\bar{\epsilon}_{i j}\right)$ is assumed for all total strain components. However, it holds only for $\delta \epsilon_{22}\left(+\bar{\epsilon}_{22}\right), \delta \epsilon_{33}\left(+\bar{\epsilon}_{33}\right)$ and $\delta \epsilon_{23}\left(+\bar{\epsilon}_{23}\right)$ at 
equilibrium.

Therefore, in the new scheme, $\sigma_{11}, \sigma_{12}$, and $\sigma_{13}$ are calculated using SAS i.e., $\sigma_{11}^{\alpha}=\sigma_{11}^{\beta}=\sigma_{11}^{S A S}, \sigma_{12}^{\alpha}=\sigma_{12}^{\beta}=\sigma_{12}^{S A S}$ and $\sigma_{13}^{\alpha}=\sigma_{13}^{\beta}=\sigma_{13}^{S A S}$. For the strain components, $\epsilon_{22}, \epsilon_{33}$, and $\epsilon_{23}$ are calculated using VTS i.e., $\epsilon_{22}^{\alpha}=\epsilon_{22}^{\beta}$ $=\epsilon_{22}^{V T S}, \epsilon_{33}^{\alpha}=\epsilon_{33}^{\beta}=\epsilon_{33}^{V T S}$ and $\epsilon_{23}^{\alpha}=\epsilon_{23}^{\beta}=\epsilon_{23}^{V T S}$. The corresponding elastic strain components are calculated as: $\epsilon_{22}^{e l, \alpha}=\epsilon_{22}^{V T S}-\epsilon_{22}^{*, \alpha}=\epsilon_{22}^{e l, V T S, \alpha}, \epsilon_{23}^{e l, \beta}=$ $\epsilon_{23}^{V T S}-\epsilon_{23}^{*, \beta}=\epsilon_{23}^{e l, V T S, \beta}$, and $\epsilon_{33}^{e l, \beta}=\epsilon_{33}^{V T S}-\epsilon_{33}^{*, \beta}=\epsilon_{33}^{e l, V T S, \beta}$

The remaining stress and strain components for each phase (the superscript $\rho$ denotes $\alpha$ or $\beta$ ) are given as:

$$
\begin{aligned}
\sigma_{22}^{\rho}= & C_{2211}^{\rho} \epsilon_{11}^{e l, \rho}+C_{2222}^{\rho} \epsilon_{22}^{e l, V T S, \rho}+C_{2233}^{\rho} \epsilon_{33}^{e l, V T S, \rho} \\
& +C_{2223}^{\rho}\left(2 \epsilon_{23}^{e l, V T S, \rho}\right)+C_{2213}^{\rho}\left(2 \epsilon_{13}^{e l, \rho}\right)+C_{2212}^{\rho}\left(2 \epsilon_{12}^{e l, \rho}\right), \\
\sigma_{33}^{\rho}= & C_{3311}^{\rho} \epsilon_{11}^{e l, \rho}+C_{3322}^{\rho} \epsilon_{22}^{e l, V T S, \rho}+C_{3333}^{\rho} \epsilon_{33}^{e l, V T S, \rho} \\
& +C_{3323}^{\rho}\left(2 \epsilon_{23}^{e l, V T S, \rho}\right)+C_{3313}^{\rho}\left(2 \epsilon_{13}^{e l, \rho}\right)+C_{3312}^{\rho}\left(2 \epsilon_{12}^{e l, \rho}\right), \\
\sigma_{23}^{\rho}= & C_{2311}^{\rho} \epsilon_{11}^{e l, \rho}+C_{2322}^{\rho} \epsilon_{22}^{e l, V T S, \rho}+C_{2333}^{\rho} \epsilon_{33}^{e l, V T S, \rho} \\
& +C_{2323}^{\rho}\left(2 \epsilon_{23}^{e l, V T S, \rho}\right)+C_{2313}^{\rho}\left(2 \epsilon_{13}^{e l, \rho}\right)+C_{2312}^{\rho}\left(2 \epsilon_{12}^{e l, \rho}\right), \\
\epsilon_{11}^{e l, \rho}= & S_{1111}^{\rho} \sigma_{11}^{S A S}+S_{1122}^{\rho} \sigma_{22}^{\rho}+S_{1133}^{\rho} \sigma_{33}^{\rho} \\
& +2 S_{1123}^{\rho} \sigma_{23}^{\rho}+2 S_{1113}^{\rho} \sigma_{13}^{S A S}+2 S_{1112}^{\rho} \sigma_{12}^{S A S}, \\
\epsilon_{13}^{e l, \rho}= & S_{1311}^{\rho} \sigma_{11}^{S A S}+S_{1322}^{\rho} \sigma_{22}^{\rho}+S_{1333}^{\rho} \sigma_{33}^{\rho} \\
& +2 S_{1323}^{\rho} \sigma_{23}^{\rho}+2 S_{1313}^{\rho} \sigma_{13}^{S A S}+2 S_{1312}^{\rho} \sigma_{12}^{S A S}, \\
\epsilon_{12}^{e l, \rho}= & S_{1211}^{\rho} \sigma_{11}^{S A S}+S_{1222}^{\rho} \sigma_{22}^{\rho}+S_{1233}^{\rho} \sigma_{33}^{\rho} \\
& +2 S_{1223}^{\rho} \sigma_{23}^{\rho}+2 S_{1213}^{\rho} \sigma_{13}^{S A S}+2 S_{1212}^{\rho} \sigma_{12}^{S A S},
\end{aligned}
$$

The unknown components $\sigma_{22}^{\rho}, \sigma_{33}^{\rho}, \sigma_{23}^{\rho}, \epsilon_{11}^{e l, \rho}, \epsilon_{12}^{e l, \rho}$ and $\epsilon_{13}^{e l, \rho}$ can be calculated in terms of the known components $\sigma_{11}^{S A S}, \sigma_{12}^{S A S}, \sigma_{13}^{S A S}, \epsilon_{22}^{e l, V T S, \rho}, \epsilon_{33}^{e l, V T S, \rho}$ and $\epsilon_{23}^{e l, V T S, \rho}$ using the above relations as given in Appendix $\mathrm{A}$.

The elastic energy is given by:

$$
\begin{aligned}
f^{e l} & =p(\phi) f^{\alpha, e l}+[1-p(\phi)] f^{\beta, e l}, \\
\text { where } f^{\rho, e l} & =\frac{1}{2}\left(\sigma_{11}^{S A S} \epsilon_{11}^{e l, \rho}+\sigma_{22}^{\rho} \epsilon_{22}^{e l, V T S, \rho}+\sigma_{33}^{\rho} \epsilon_{33}^{e l, V T S, \rho}\right),
\end{aligned}
$$

The driving force due to the elastic energy is calculated as:

$$
\frac{\partial f^{e l}}{\partial \phi}=p^{\prime}(\phi)\left(f^{\alpha, e l}-f^{\beta, e l}\right)+p(\phi) \frac{\partial f^{\alpha, e l}}{\partial \phi}+[1-p(\phi)] \frac{\partial f^{\beta, e l}}{\partial \phi},
$$


where

$$
\begin{aligned}
\frac{\partial f^{\rho, e l}}{\partial \phi}= & \frac{1}{2}\left[\frac{\partial \sigma_{11}^{S A S}}{\partial \phi} \epsilon_{11}^{e l, \rho}+\sigma_{11}^{S A S} \frac{\partial \epsilon_{11}^{e l, \rho}}{\partial \phi}+\frac{\partial \sigma_{22}^{\rho}}{\partial \phi} \epsilon_{22}^{e l, V T S, \rho}+\frac{\partial \sigma_{33}^{\rho}}{\partial \phi} \epsilon_{33}^{e l, V T S, \rho}+2 \frac{\partial \sigma_{23}^{\rho}}{\partial \phi} \epsilon_{23}^{e l, V T S, \rho}\right] \\
& +\frac{1}{2}\left[2 \frac{\partial \sigma_{13}^{S A S}}{\partial \phi} \epsilon_{13}^{e l, \rho}+2 \sigma_{13}^{S A S} \frac{\partial \epsilon_{13}^{e l, \rho}}{\partial \phi}+2 \frac{\partial \sigma_{12}^{S A S}}{\partial \phi} \epsilon_{12}^{e l, \rho}+2 \sigma_{12}^{S A S} \frac{\partial \epsilon_{12}^{e l, \rho}}{\partial \phi}\right],
\end{aligned}
$$

The stress and strain derivatives are calculated as given in Appendix B. A reduced version of this model in $2 \mathrm{D}$ is given in Appendix $\mathrm{C}$.

\subsection{Non-planar interfaces}

As discussed in our previous article, for non-planar interfaces, a local reference frame is chosen with coordinate axes normal and tangential to the interface and all tensors are transformed to this reference frame. This means that for every point in the interface with non-zero interface curvature, a different local reference frame is chosen, as shown in Fig. 5(a). The unit

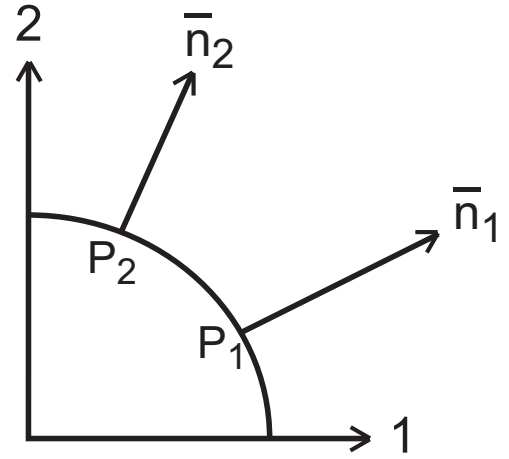

(a)

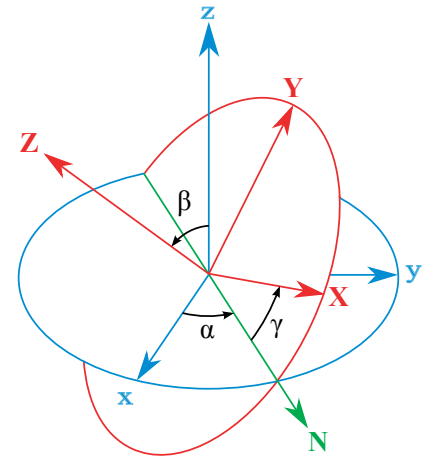

(b)

Figure 5: (a) For implementing the new scheme at non-planar interfaces, local reference frames are chosen based on the location of each point on the interface. For instance, for point $\mathrm{P}_{1}$, the normal direction $\mathrm{n}_{1}$ is chosen as the new principal axis and for $\mathrm{P}_{2}$, it is $\mathrm{n}_{2}$. (b) In 3D [20], a local reference frame (XYZ) is derived from the original reference frame (xyz) at every point on the interface, with $\mathrm{X}$ being the direction of the normal to the interface.

normal at every point is given by $\hat{n}=\frac{\vec{\nabla} \phi}{|\vec{\nabla} \phi|}$. In each of these new reference frames, the interfacial conditions described in Section 2 are valid, i.e., the stress components $\sigma_{11}^{\prime}, \sigma_{12}^{\prime}$, and $\sigma_{13}^{\prime}$ are equal in the two phases and the strain 
components $\delta \epsilon_{22}^{\prime}, \delta \epsilon_{33}^{\prime}$ and $\delta \epsilon_{23}^{\prime}$ are equal in the two phases, where these are the values in the new reference frame transformed from the old one according to:

$$
\begin{aligned}
\sigma_{i j}^{\prime} & =a_{i m} a_{j n} \sigma_{m n}, \\
\epsilon_{i j}^{\prime} & =a_{i m} a_{j n} \epsilon_{m n}, \\
C_{i j k l}^{\prime} & =a_{i m} a_{j n} a_{k o} a_{l p} C_{m n o p}, \\
S_{i j k l}^{\prime} & =a_{i m} a_{j n} a_{k o} a_{l p} S_{m n o p} .
\end{aligned}
$$

Here, $a_{i j}$ is the transformation matrix (not a tensor). In $2 \mathrm{D}, a_{11}=\cos \theta, a_{22}$ $=\cos \theta, a_{12}=-\sin \theta$ and $a_{11}=\sin \theta$, where $\theta$ is the angle of rotation of the new reference frame with respect to the original reference frame.

In $3 \mathrm{D}$, at every point in the interface, the new reference frame (XYZ) is obtained from the original one (xyz) as shown in Fig. 5(b). Here, X corresponds to the direction of the normal to the interface. The corresponding transformation matrix is given by:

$a_{i j}=\left(\begin{array}{ccc}\cos \beta \cos \gamma & \cos \alpha \sin \gamma+\sin \alpha \sin \beta \cos \gamma & \sin \alpha \sin \gamma-\cos \alpha \sin \beta \cos \gamma \\ -\cos \beta \sin \gamma & \cos \alpha \cos \gamma-\sin \alpha \sin \beta \sin \gamma & \sin \alpha \cos \gamma+\cos \alpha \sin \beta \sin \gamma \\ \sin \beta & -\sin \alpha \cos \beta & \cos \alpha \cos \beta\end{array}\right)$,

where $\alpha, \beta$ and $\gamma$ are the Euler angles of rotation.

For a general system with arbitrary interface curvatures, the new scheme is implemented as follows. Firstly, mechanical equilibrium is solved using SAS and VTS in the original reference frame. The stresses $\sigma_{i j}^{S A S}$, the strains $\epsilon_{i j}^{e l, V T S}$, and the stiffnesses are transformed to the new local reference frame. Then, equations (40) - (45) are applied and the quantities $\sigma_{22}^{\rho}, \sigma_{33}^{\rho}, \sigma_{23}^{\rho}, \epsilon_{11}^{e l, \rho}$, $\epsilon_{12}^{e l, \rho}$ and $\epsilon_{13}^{e l, \rho}$ are calculated in the new reference frame. Using these, the elastic energy and the driving force $\frac{\partial f^{e l}}{\partial \phi}$ are computed using (46) and (47) respectively in order to solve the phase-field equation (32).

\section{Simulation results and discussion}

The aim of the simulations is to validate the new scheme described above for systems with shear and applied strains, and non-planar interfaces. Therefore, the following geometries for which analytical solutions exist are considered in the simulations: 2D inhomogeneous systems with planar and nonplanar interfaces and a 3D inhomogeneous system with planar interface. In 
the bulk of the two phases, the strains generated, elastic energy, and change in equilibrium composition are compared with the analytical solutions. At the interface, the excess interfacial energies for different interface widths are analysed.

\subsection{Implementation}

In this study, the system is constrained such that the macroscopic deformation is given by a constant applied strain value, $\overline{\epsilon_{k l}}=\overline{\epsilon_{k l}^{a}}$. When there are no externally applied strains, $\overline{\epsilon_{k l}^{a}}=0$. The stresses and strains at mechanical equilibrium are calculated after solving the displacement field (as explained in Section 3) using the spectral iterative perturbation method of $\mathrm{Hu}$ and Chen [21]. The driving forces for phase evolution from the chemical, interfacial and elastic contributions are calculated as formulated in Sections 3 and 4 . The phase-field equations are solved at every time step using finite difference discretisation.

\subsection{Simulation setup}

The following parameters are used for all simulations unless otherwise mentioned. Chemical energy densities of the two phases are defined by the parameters $A^{\alpha}=A^{\beta}=10^{5}, c_{\alpha, 0}=0.2$, and $c_{\beta, 0}=0.8$. The kinetic parameters are taken as $\mathrm{L}=10^{-7}, \mathrm{M}=10^{-10}$. These do not correspond to physical values. Simulations are performed starting with a sharp interface in the different configurations and initial compositions $c_{\alpha, 0}$ and $c_{\beta, 0}$ in $\alpha$ and $\beta$ respectively. All simulations are performed until equilibrium is reached, i.e., until the interface becomes fully diffuse and the composition and phase-field variables do not change significantly any further.

\subsection{Planar interface}

5.3.1. Input parameters for $2 D$ systems

A 2 D rectangular system of size $4 \times 128$ grid points with equal areas of the two phases is considered as shown in Fig. 1(a). The grid size is $\Delta \mathrm{x}=$ 0.001 and time step $\Delta \mathrm{t}=0.05$. Eigenstrains are taken as $\epsilon_{11}^{*, \beta}=\epsilon_{22}^{*, \beta}=0.02$. There are no applied strains for the simulations in Section 5.3.2. For the simulations presented in Section 5.3.3, the applied strain is given by: $\overline{\epsilon_{k l}^{a}}=$ $\left[\begin{array}{lll}0.01 & 0 & 0\end{array}\right]$. The stiffness tensors for $\alpha$ and $\beta$ in their reduced $2 \mathrm{D}$ forms are 
assumed to be:

$$
\begin{aligned}
& C_{i j k l}^{\alpha}=10^{3}\left(\begin{array}{ccc}
168.57 & 69.11 & 67.15 \\
69.11 & 165.39 & 67.153 \\
67.153 & 67.153 & 45.981
\end{array}\right), \\
& C_{i j k l}^{\beta}=10^{3}\left(\begin{array}{lll}
82.74 & 57.85 & 34.21 \\
57.85 & 82.74 & 34.21 \\
34.21 & 34.21 & 28.18
\end{array}\right) .
\end{aligned}
$$

The order of magnitude of these values do not correspond to a specific physical system. They are so chosen only in order to speed up the simulations. It was verified in our previous article [1] that the correctness of the model and the contribution to excess interfacial properties are not affected by the magnitude of these parameters.

Simulations are performed for two different widths with the same interfacial energy. The parameters $\mathrm{W}=500$ and $\kappa=0.01$ give interfacial energy $\left(\sigma_{\text {int }}\right)=0.527$ and interfacial width $\left(l_{\text {int }}\right)=13.9 \Delta \mathrm{x}$ (referred to as Case w1 henceforth). For the second instance (Case w2), the parameters $\mathrm{W}=750$ and $\kappa=0.0067$ give $\sigma_{\text {int }}=0.527$ and $l_{\text {int }}=9.3 \Delta \mathrm{x}$. Also, in order to study the effect of interfacial excess quantities on the absolute value of the interface width, simulations are performed at a different length scale (Case g2) with grid size $\Delta \mathrm{x}=0.01, \mathrm{~W}=7.5$ and $\kappa=0.0667$ giving $l_{\text {int }}=9.3 \Delta \mathrm{x}$ and $\sigma_{\text {int }}$ $=0.527$, and all the other parameters remaining the same.

\subsubsection{Misfit between phases}

The composition and elastic energy profiles obtained at equilibrium given in Figs. 6(a) and 6(b) confirm that the change in interface width does not affect the bulk properties. The bulk elastic energy is constant inside the two phases (Fig. 6(b)). Therefore, if the elastic energy formulation does not lead to excess interfacial energy, the elastic energy density should interpolate linearly with the interpolation function $p(\phi)$. This is so for all cases as shown in Fig. 6(c). More details about the calculation of interfacial excess energy are provided in our previous article. The stress profiles overlap for all cases as shown in Fig. 6(d).

The heterogeneous strains generated, the change in composition, the elastic energies in the two phases and the excess elastic energy are listed in Table 1 for the three cases. The analytical solutions are calculated using the relations given in 2.2.1 and Section 2.2.3. The compositions from the simulations 


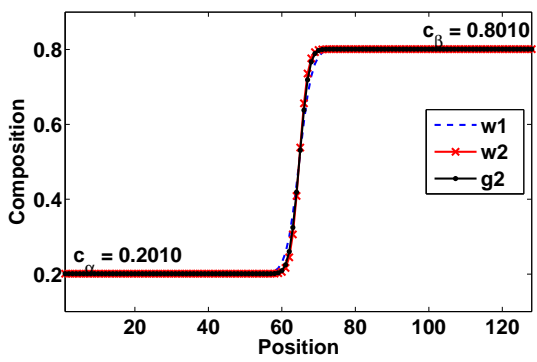

(a)

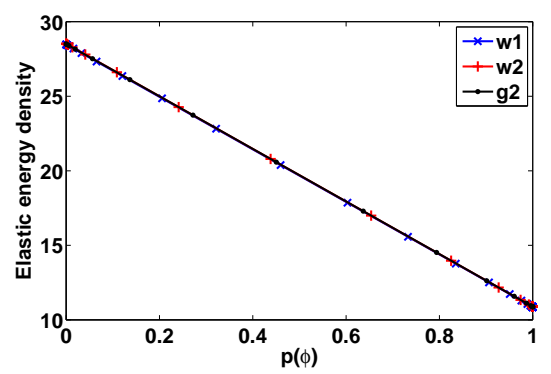

(c)

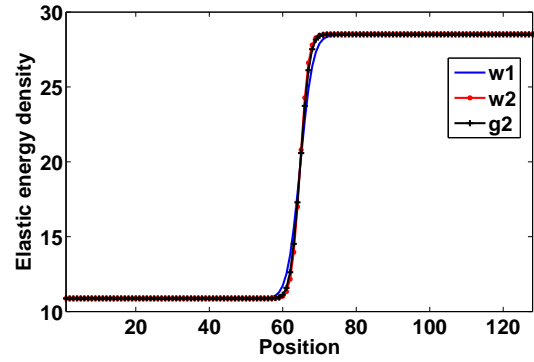

(b)

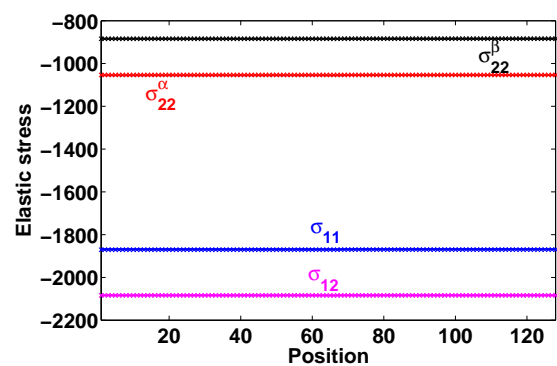

(d)

Figure 6: Comparison of the bulk properties obtained from 2D phase-field simulations with shear strains for three different interface widths: (a) Composition and (b) elastic energy densities are constant inside the two phases and are consistent with the analytical results. (c) Elastic energy density vs. $\mathrm{p}(\phi)$ is linear for all cases, proving the absence of excess interfacial energy. (d) The profiles of the different stress components overlap for different interface widths (same markers used as before).

follow the analytical solution. The heterogeneous strain component $\delta \epsilon_{22}$ is zero in both phases in the simulations, which follows the analytical solution and hence not listed in the table. The excess elastic energy density $f_{x s}^{e l}(\%)$ is calculated as the percentage of difference between the simulated energy density for the diffuse interface and the energy density for the same system with a sharp interface. The elastic energy densities agree well with the analytical results and are within $1 \%$ of the calculated values. There are no significant differences with change in interface width (Case w2 and Case g2). The difference in the heterogeneous strains $(5 \%)$ is only due to limited size and change in the interface position due to strain-induced diffusion. In order to further examine this, simulations were performed for a larger system size of $2 \times 512$ gridpoints for two different interface widths as before (512-Case $\mathrm{w} 1$ and 512-Case w2). The strains generated are now within $2.5 \%$ of the ana- 
lytical values and again, for two different interface widths, the excess energy generated is insignificant. Moreover, the difference in elastic energy densities for different interface widths is also insignificant, so we can conclude that the results do not depend on the interface width.

Table 1: Bulk properties obtained from 2D simulations $(4 \times 128$ system size $)$ with misfit between phases performed for different interface widths: Compositions from simulations agree with the analytical values; there is a small error in the other quantities, but this is mainly due to the limited system size. A larger system $(2 \times 512)$ improves the accuracy. For all cases, change in interface width does not give rise to excess elastic energy.

\begin{tabular}{ccccccc}
\hline Property & Analytical & Case w1 & Case w2 & Case g2 & 512-Case w1 & 512-Case w2 \\
\hline$\delta \epsilon_{11}^{\alpha}$ & -0.0083 & -0.0082 & -0.0082 & -0.0082 & -0.0083 & -0.0083 \\
$\delta \epsilon_{11}^{\beta}$ & 0.0083 & 0.0087 & 0.0086 & 0.0086 & 0.0085 & 0.0084 \\
$2 \delta \epsilon_{12}^{\alpha}$ & -0.0070 & -0.0073 & -0.0072 & -0.0073 & -0.0071 & -0.0071 \\
$2 \delta \epsilon_{12}^{\beta}$ & 0.0070 & 0.0066 & 0.0068 & 0.0067 & 0.0070 & 0.0070 \\
$c_{\alpha}^{e}$ & 0.2010 & 0.2010 & 0.2010 & 0.2010 & 0.2010 & 0.2010 \\
$c_{\beta}^{e}$ & 0.8010 & 0.8010 & 0.8010 & 0.8010 & 0.8010 & 0.8010 \\
$f_{\alpha}^{e l}$ & 10.96 & 10.86 & 10.88 & 10.88 & 10.90 & 10.90 \\
$f_{\beta}^{e l}$ & 28.72 & 28.47 & 28.52 & 28.51 & 28.58 & 28.60 \\
$f_{x s}^{e l}(\%)$ & 0 & -0.1498 & -0.1506 & -0.1482 & -0.1518 & -0.1511 \\
\hline
\end{tabular}

\subsubsection{Misfit between phases and applied strains}

We consider the same properties as before but with an externally applied tensile strain $\left(\overline{\epsilon_{11}}=0.01\right)$. The bulk properties change significantly compared to the previous case with no applied strains, as listed in Table 2.

The profiles of compositon and elastic energy density in Fig. 7(a) and 7(b) confirm the good agreement with the analytical results. Even with applied strains, the elastic energy density interpolates linearly for different interface widths as shown in Fig. 7(c). There is no significant effect of the change in interface width on the bulk properties of the two phases and the (insignificant) excess elastic energy.

\subsubsection{D inhomogeneous system with planar interface and misfit between phases}

A 3 D cuboidal system of size $4 \times 128 \times 4$ grid points with equal volumes of the two phases is considered in the configuration given in Fig. 2(a). The grid size is $\Delta \mathrm{x}=0.1$ and time step $\Delta \mathrm{t}=1$. The eigenstrain in the $\beta$ phase is 


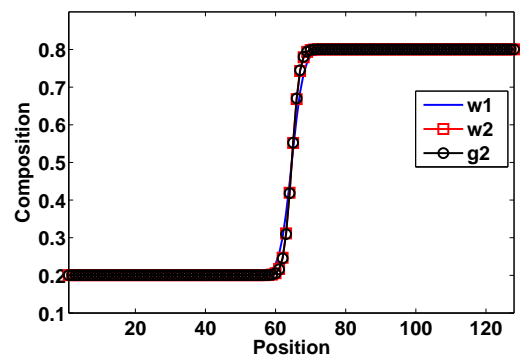

(a)

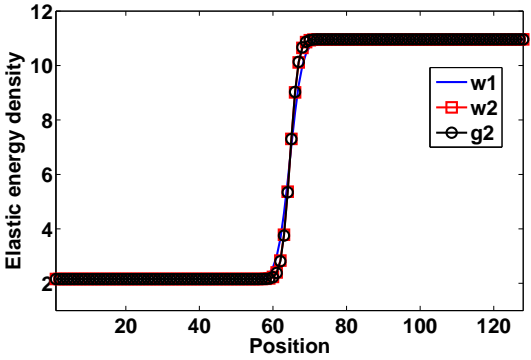

(b)

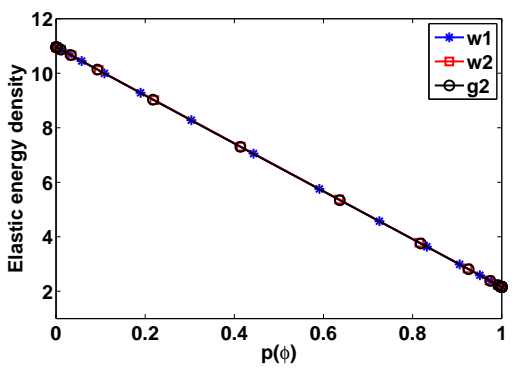

(c)

Figure 7: Comparison of the bulk properties obtained from 2D phase-field simulations with misfit between phases and applied strains for three different interface widths: (a) Composition profiles and (b) elastic energy density profiles are consistent with analytical results. (c) Elastic energy density interpolates linearly with $\mathrm{p}(\phi)$, proving that the model is quantitative.

taken as $\epsilon_{k l}^{*, \beta}=\left[\begin{array}{llllll}0.01 & 0.01 & 0.01 & 0.01 & 0.01 & 0.01\end{array}\right]$. There are no applied strains. 
Table 2: Bulk properties obtained from 2D simulations with misfit between phases and applied strains performed with different interface widths: Compositions and elastic energies from simulations agree with the analytical values. There is a small error in the strains due to the finite system size. Change in interface width does not change the bulk or interfacial properties significantly.

\begin{tabular}{ccccc}
\hline Property & Analytical & Case w1 & Case w2 & Case g2 \\
\hline$\delta \epsilon_{11}^{\alpha}$ & -0.0120 & -0.0118 & -0.0119 & -0.0119 \\
$\delta \epsilon_{11}^{\beta}$ & 0.0120 & 0.0122 & 0.0121 & 0.0121 \\
$2 \delta \epsilon_{12}^{\alpha}$ & -0.0065 & -0.0068 & -0.0067 & -0.0067 \\
$2 \delta \epsilon_{12}^{\beta}$ & 0.0065 & 0.0062 & 0.0063 & 0.0063 \\
$c_{\alpha}^{e}$ & 0.2005 & 0.2005 & 0.2005 & 0.2005 \\
$c_{\beta}^{e}$ & 0.8005 & 0.8005 & 0.8005 & 0.8005 \\
$f_{\alpha}^{e l}$ & 2.17 & 2.16 & 2.16 & 2.16 \\
$f_{\beta}^{e l}$ & 11.00 & 10.95 & 10.96 & 10.96 \\
$f_{x s}^{e l}(\%)$ & 0 & -0.1244 & -0.1236 & -0.1301 \\
\hline
\end{tabular}

The stiffness tensors for $\alpha$ and $\beta$ are assumed to be:

$$
\begin{aligned}
& C_{i j k l}^{\alpha}=\left(\begin{array}{cccccc}
176.3 & 112.6 & 112.6 & 0 & 0 & 0 \\
112.6 & 176.3 & 112.6 & 0 & 0 & 0 \\
112.6 & 112.6 & 176.3 & 0 & 0 & 0 \\
0 & 0 & 0 & 93.9 & 0 & 0 \\
0 & 0 & 0 & 0 & 93.9 & 0 \\
0 & 0 & 0 & 0 & 0 & 93.9
\end{array}\right), \\
& C_{i j k l}^{\beta}=\left(\begin{array}{cccccc}
158.4 & 81.8 & 86.3 & 0 & 0 & 0 \\
81.8 & 183.3 & 101.4 & 0 & 0 & 0 \\
86.3 & 101.4 & 128.2 & 0 & 0 & 0 \\
0 & 0 & 0 & 56.8 & 0 & 0 \\
0 & 0 & 0 & 0 & 52.0 & 0 \\
0 & 0 & 0 & 0 & 0 & 36.2
\end{array}\right) \text {. }
\end{aligned}
$$

The parameters $\mathrm{W}=6.6$ and $\kappa=0.68182$ give interfacial energy $\sigma_{\text {int }}=$ 0.5 and interfacial width $l_{\text {int }}=10 \Delta \mathrm{x}$. The chemical energy is given by the parameters $A^{\alpha}=A^{\beta}=10^{3}, c_{\alpha, 0}=0.2$, and $c_{\beta, 0}=0.8$. The kinetic parameters are taken as $\mathrm{L}=10^{-5}$ and $\mathrm{M}=10^{-7}$.

Simulations are performed to test the results with change in interface width. Therefore, two more studies are performed: one (w2) with interfacial 
width $=15 \Delta \mathrm{x}$ while keeping the interfacial energy same, and the other (g2) with a different grid size $\Delta \mathrm{x}=1$, time step $\Delta \mathrm{t}=100$, and interface width $=10 \Delta \mathrm{x}$.

The composition and the elastic energy in the two phases are independent of the interface width as shown in Figs. 8(a) and 8(b). The elastic energy varies linearly with the interpolation function for all three cases, as shown in Fig. 8(c). This proves that there is no excess contribution to the interfacial energy.

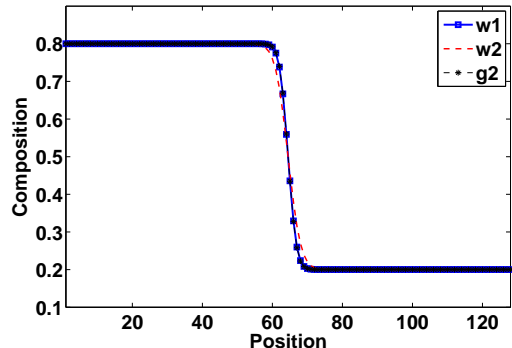

(a)

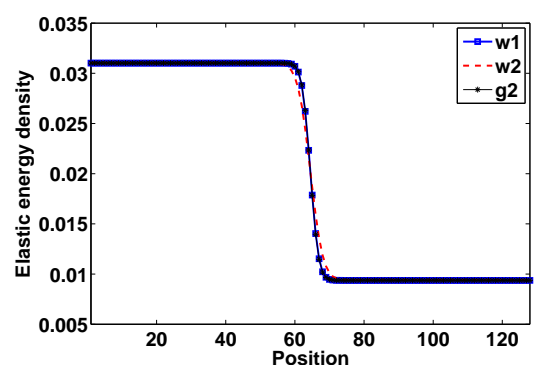

(b)

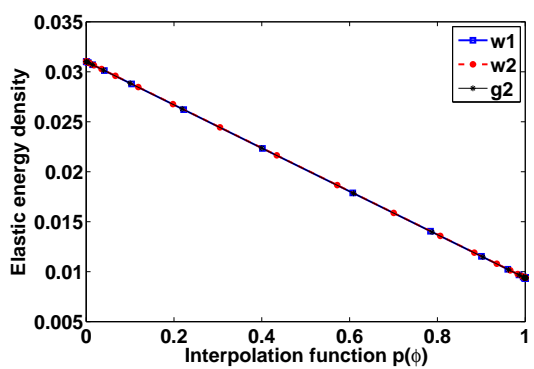

(c)

Figure 8: Comparison of the bulk properties obtained from 3D phase-field simulations with misfit between phases for three different interface widths: (a) Composition profiles and (b) Elastic energy density profiles are consistent with analytical results. (c) Elastic energy density interpolates linearly with $\mathrm{p}(\phi)$ for all cases.

The heterogeneous strains generated are calculated analytically using the relations given in Section 2.2.2 and the new equilibrium compositions using the equations given in Section 2.2.3. The bulk properties in the two phases computed from the simulations are listed along with the analytical values in Table 3. The simulation results are in excellent agreement with the analytical solutions. No interfacial excess energy is generated for all interface widths. 
Table 3: Bulk properties obtained from 3D simulations with misfit between phases performed with different interface widths: The bulk properties are in excellent agreement with the analytical solution. No interfacial excess energy is generated for all cases.

\begin{tabular}{ccccc}
\hline Property & Analytical & Case w1 & Case w2 & Case g2 \\
\hline$\delta \epsilon_{11}^{\alpha}$ & -0.009755 & -0.009769 & -0.009778 & -0.009769 \\
$\delta \epsilon_{11}^{\beta}$ & 0.009755 & 0.009739 & 0.009729 & 0.009739 \\
$2 \delta \epsilon_{12}^{\alpha}$ & -0.002782 & -0.002782 & -0.002782 & -0.002782 \\
$2 \delta \epsilon_{12}^{\beta}$ & 0.002782 & 0.002784 & 0.002784 & 0.002784 \\
$2 \delta \epsilon_{13}^{\alpha}$ & -0.003564 & -0.003563 & -0.003563 & -0.003563 \\
$2 \delta \epsilon_{13}^{\beta}$ & 0.003564 & 0.003566 & 0.003566 & 0.003566 \\
$c_{\alpha}^{e}$ & 0.2001 & 0.2001 & 0.2001 & 0.2001 \\
$c_{\beta}^{e}$ & 0.8001 & 0.8001 & 0.8001 & 0.8001 \\
$f_{\alpha}^{e l}$ & 0.009348 & 0.009372 & 0.009388 & 0.009372 \\
$f_{\beta}^{e l}$ & 0.03099 & 0.03102 & 0.03103 & 0.03102 \\
$f_{x s}^{e l}(\%)$ & 0 & -0.01813 & -0.01814 & -0.01814 \\
\hline
\end{tabular}

5.4. 2D system with an elliptical precipitate in a matrix (non-planar interface)

A 2 D system of size $128 \times 128$ grid points with an elliptical precipitate $(\beta)(\mathrm{a}=15, \mathrm{~b}=10)$ at the centre is considered as shown in Fig. 3(a). The matrix phase $(\alpha)$ is taken as the reference state. Eigenstrains in the precipitate are taken as $\epsilon_{11}^{*, \beta}=\epsilon_{22}^{*, \beta}=0.02$ (zero shear component). There are no applied strains. The stiffness tensors for $\alpha$ and $\beta$ are taken as:

$$
\begin{aligned}
C_{i j k l}^{\alpha} & =10^{3}\left(\begin{array}{ccc}
79 & 27.77 & 0 \\
27.77 & 79 & 0 ; \\
0 & 0 & 25.62
\end{array}\right), \\
C_{i j k l}^{\beta} & =10^{3}\left(\begin{array}{ccc}
132.44 & 60.64 & 0 \\
60.64 & 132.44 & 0 \\
0 & 0 & 35.9
\end{array}\right) .
\end{aligned}
$$

The grid size is taken as $\Delta \mathrm{x}=0.001$ and time step $\Delta \mathrm{t}=0.01$. The parameters $\mathrm{W}=1250$ and $\kappa=0.004$ give $\sigma_{\text {int }}=0.527$ and $l_{\text {int }}=5.6 \Delta \mathrm{x}$ (Case 128-w1). We also study this system at another length scale with grid size $\Delta \mathrm{x}=0.01$ and $\Delta \mathrm{t}=0.1$ (Case 128-g2). For that case, the parameters $\mathrm{W}=125$ and $\kappa$ $=0.04$ give $\sigma_{\text {int }}=0.527$ and $l_{\text {int }}=5.6 \Delta \mathrm{x}$. 
The analytical solution for this case given by Jin et al. [15] is described in Sec. 2.3. The strains and stresses remain constant inside the precipitate. The strains and stresses (and thereby elastic energy) in the matrix tend to zero with increasing distance away from the centre of the ellipse. Since the elastic energy is not constant in the two phases, the absence of excess interfacial energy cannot be shown by plotting the elastic energy density as a function of $p(\phi)$. It does not result in a linear plot. In order to prove that the scheme is quantitative, the difference in the total elastic energy between the analytical and simulated solutions are compared in the case of different interface widths since any excess contribution to the interfacial energy can result only from the elastic energy formulation which has not yet fully been tested to be quantitative. If there is excess interfacial energy, then the total (elastic) energy changes with the interface width. In the absence of excess interfacial energy, same accuracy (for the bulk properties) is expected for same number of grid points across the interface irrespective of the grid spacing, i. e. , we expect similar results for Cases 128-w1 and 128-g2.

Fig. 9 gives the elastic energy, stress and strain plots along a horizontal line starting at the centre of the ellipse and passing through the semi-major axis till the edge of the system. The first four columns of Table 4 give the bulk properties from simulations and the analytical solution. The numbers in brackets for the 'deviation' of the results of the simulations indicate the percentage difference between the simulated and analytical values. The second number for 'Deviation' for case g2 indicates the percentage difference between the case w1 and case g2 results.

Firstly, the trends in stresses, strains, and elastic energies follow those from the analytical solution. The variation of $\sigma_{11}$ given in Fig. 9(a) shows that it is constant inside the precipitate and falls off away from the ellipse as expected from the analytical solution. The $\sigma_{22}$ component shows a discontinuity and $\sigma_{12}$ is equal to zero (Fig. 9(b)), also as expected. The elastic strains (Fig. 9(d)) follow the analytical solution closely except near the interface. Fig. 9(c) shows that there is a large difference $(17-18 \%)$ between the elastic energies in the precipitate from the analytical solution and the simulations. However, the total elastic energy density is very close $(1-2 \%)$ to the analytical solution. The stresses and strains also follow a similar trend. They do not attain the peak values at the interface due to the limitation in the number of grid points at the interface and the grid spacing. If we, however, look at the difference in the bulk values with change in interface width but with the same number of grid points at the interface (between 
Cases 128-w1 and 128-g2), we see that there is only $1-4 \%$ difference for all properties. This is an important outcome of these simulations and will be further examined through simulations with a larger system size that are discussed later in this section.

The composition change in the matrix and precipitate at the interface is given in Table 4. Apart from the effect of strains, the interface curvature also shifts the equilibrium composition as given by (21) and (22). The composition change is calculated assuming an 'effective circle radius' for the ellipse. This, along with the fact that the stresses and strains of the matrix at the interface do not attain the peak value, causes the deviation in the values from simulations compared to the analytical solution. For Case 128-g2, due to different grid spacing, the composition change is also different compared to Case 128-w1 and this new analytically calculated value is indicated in brackets below the value obtained from simulations.

There are several factors which affect the solutions from the simulations in the particle and in the matrix. Firstly, with the limited system size, the stresses and strains in the matrix do not tend to zero far away from the ellipse. The analytical solutions, on the other hand, assume that the matrix is infinite. Next, with only 5.6 grid points to resolve the interface $\left(l_{\text {int }}=\right.$ $5.6 \Delta \mathrm{x})$, the resolution may not be large enough to capture the jump in properties at the interface. It has been shown even for other schemes [4, 22] that generally, greater accuracy in the bulk properties can be achieved by using a smaller grid spacing, thereby increasing the total number of grid points also. Knowing this, in this article, we focus on how a change in interface width, while keeping the same number of grid points across an interface, affects the results. For the first set of simulations with $128 \times 128$ gridpoints, we showed that the effects of changing the length scale of the system (case g2), which changes the 'absolute' value of the interface width, are small. Next, we will take up a larger system with a larger precipitate so that we can have a larger interface width and study the effects of changing the interface width on the bulk properties. We will also study the effect of changing the interface width by making the interface more diffuse (increasing the number of grid points corresponding to the interface region) but keeping the grid spacing and total system size constant. This will help show that in the absence of excess interfacial energy, the total energy at the interface will only 'spread out' and not increase when there is a change in the interface width.

For a larger system size with $256 \times 256$ grid points, which is closer to 


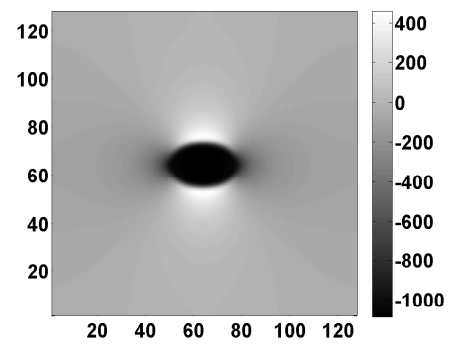

(a)

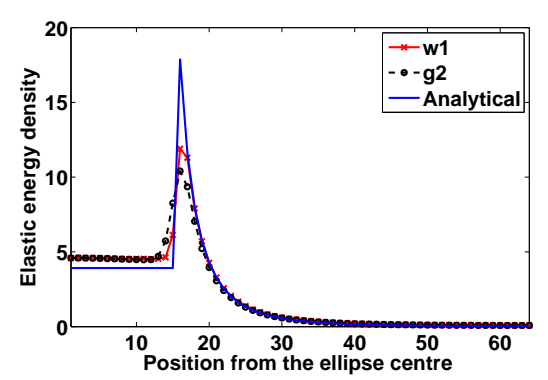

(c)

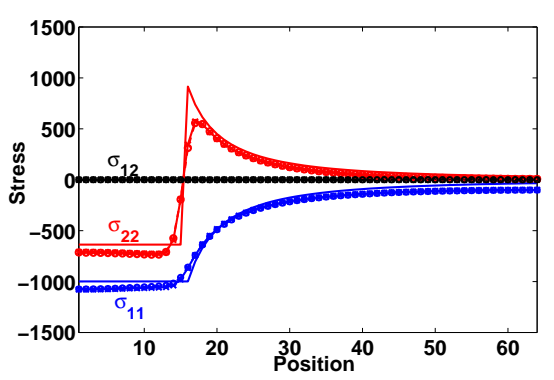

(b)

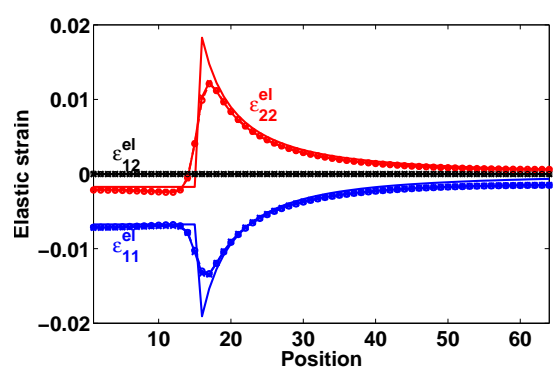

(d)

Figure 9: Simulation results for a 2D system with an elliptical precipitate in a matrix of size $128 \times 128$ grid points: (a) $\sigma_{11}$ field shows that it is constant inside the precipitate as expected and reduces as we move away from the precipitate. (b) $\sigma_{11}$ and $\sigma_{12}$ are continuous at the interface and $\sigma_{22}$ is not continuous, as expected. (c) Elastic energy in the precipitate varies upto $30 \%$ from the analytical value but the profiles follow the general trend of the analytical solution. (d) The elastic strain profiles agree well with the analytical profiles except near the interface. There are significant differences between the analytical and simulated profiles for all quantities due to the resolution at the interface and limited system size. (Same markers are used to indicate the stress and strain profiles for the different cases as those used in the elastic energy density profiles (Fig. 9(c)).)

the infinite matrix assumption than $128 \times 128$ grid points, an elliptical precipitate with axes $\mathrm{a}=25$ and $\mathrm{b}=20$ is considered so that a larger interface width can be taken. This ensures that a larger area of the precipitate has a value of $\phi=0$, i.e., does not remain in the diffuse interface region and the accuracy in the bulk properties is expected to improve. The following parameters are used for this set of simulations: $\Delta \mathrm{x}=1, \Delta \mathrm{t}=100, A^{\alpha}=$ $A^{\beta}=1000, c_{\alpha, 0}=0.2, c_{\beta, 0}=0.8, C_{1111}^{\alpha}=C_{2222}^{\alpha}=79, C_{1122}^{\alpha}=27.77, C_{1212}^{\alpha}$ $=25.62, C_{1111}^{\beta}=C_{2222}^{\alpha}=132.44, C_{1122}^{\alpha}=60.64, C_{1212}^{\alpha}=35.9, \epsilon_{11}^{*, \beta}=\epsilon_{22}^{*, \beta}=$ 0.02 . 
Table 4: Bulk properties obtained from 2D simulations with an elliptical precipitate for different interface widths and system sizes $(128 \times 128$ and $256 \times 256)$ : The first number in brackets for 'Deviation' is the percentage difference with respect to the analytical value and the second number is the percentage difference with respect to the simulated value for Case w1. The deviation from analytical results is due to the limited system size and grid spacing. The main conclusion is that for the same number of grid points and system size, the same accuracy is attained at different grid spacings (Cases 128-w1 and 128-g2, Cases 256-w1-25 and 256-g2-25). Also, for the same system size and grid spacing, making the interface more diffuse does not affect the bulk properties (Cases 256-w1-25 and 256-w2-25) much. Excess energy is not generated due to the change in interface width as seen from the very good agreement in the total elastic energy density of the system with the analytical solution for all cases.

\begin{tabular}{|c|c|c|c|c|c|c|c|}
\hline Property & $\begin{array}{c}\text { Analytical } \\
128-15\end{array}$ & 128-w1 & 128-g2 & $\begin{array}{c}\text { Analytical } \\
256-25\end{array}$ & 256-w1-25 & $256-w 2-25$ & 256-g2-25 \\
\hline $\begin{array}{c}\sigma_{11}^{\beta} \\
\text { Deviation }\end{array}$ & -998.4 & $\begin{array}{l}-1078 \\
(8.0 \%)\end{array}$ & $\begin{array}{c}-1073 \\
(7.5 \%,-0.46 \%)\end{array}$ & -0.9122 & $\begin{array}{c}-0.9814 \\
(7.6 \%)\end{array}$ & $\begin{array}{c}-0.9846 \\
(7.9 \%, 0.33 \%)\end{array}$ & $\begin{array}{c}-0.9760 \\
(7.0 \%,-0.55 \%)\end{array}$ \\
\hline $\begin{array}{c}\sigma_{22}^{\beta} \\
\text { Deviation }\end{array}$ & -637.7 & $\begin{array}{l}-710.4 \\
(11 \%)\end{array}$ & $\begin{array}{c}-714.0 \\
(12 \%, 0.51 \%)\end{array}$ & -0.7126 & $\begin{array}{c}-0.7699 \\
(8.0 \%)\end{array}$ & $\begin{array}{c}-0.7776 \\
(9.1 \%, 1.0 \%)\end{array}$ & $\begin{array}{c}-0.7749 \\
(8.7 \%, 0.65 \%)\end{array}$ \\
\hline $\begin{array}{c}\epsilon_{11}^{e l, \beta} \\
\text { Deviation }\end{array}$ & -0.006749 & $\begin{array}{c}-0.007192 \\
(6.6 \%)\end{array}$ & $\begin{array}{c}-0.007138 \\
(5.8 \%,-0.75 \%)\end{array}$ & -0.005597 & $\begin{array}{c}-0.006000 \\
(7.2 \%)\end{array}$ & $\begin{array}{c}-0.006005 \\
(7.3 \%, 0.083 \%)\end{array}$ & $\begin{array}{c}-0.005932 \\
(6 \%,-1.1 \%)\end{array}$ \\
\hline $\begin{array}{c}\epsilon_{22}^{e l, \beta} \\
\text { Deviation }\end{array}$ & -0.001725 & $\begin{array}{c}-0.002071 \\
(20 \%)\end{array}$ & $\begin{array}{c}-0.002146 \\
(24 \%, 3.6 \%)\end{array}$ & -0.002818 & $\begin{array}{c}-0.003107 \\
(10 \%)\end{array}$ & $\begin{array}{c}-0.003122 \\
(11 \%, 0.48 \%)\end{array}$ & $\begin{array}{l}-0.003172 \\
(12 \%, 2 \%)\end{array}$ \\
\hline $\begin{array}{c}f_{\beta}^{e l} \\
\text { Deviation }\end{array}$ & 3.919 & $\begin{array}{l}4.613 \\
(18 \%)\end{array}$ & $\begin{array}{c}4.598 \\
(17 \%,-0.32 \%)\end{array}$ & 0.003557 & $\begin{array}{c}0.004140 \\
(16 \%)\end{array}$ & $\begin{array}{c}0.004170 \\
(17 \%, 0.72 \%)\end{array}$ & $\begin{array}{c}0.004124 \\
(16 \%,-0.39 \%)\end{array}$ \\
\hline $\begin{array}{c}f_{\text {total }}^{e l} \\
\text { Deviation }\end{array}$ & 7668 & $\begin{array}{c}7777 \\
(1.4 \%)\end{array}$ & $\begin{array}{c}7491 \\
(-2.3 \%,-3.7 \%)\end{array}$ & 25.24 & $\begin{array}{c}25.57 \\
(1.3 \%)\end{array}$ & $\begin{array}{c}25.34 \\
(0.40 \%,-0.90 \%)\end{array}$ & $\begin{array}{c}25.29 \\
(0.20 \%,-1.1 \%)\end{array}$ \\
\hline$c_{\alpha}^{e}$ & 0.2010 & 0.2007 & $\begin{array}{c}0.2004 \\
(0.2004 \text { anal. })\end{array}$ & 0.20007 & 0.20007 & 0.20005 & $\begin{array}{c}0.20034 \\
(0.20040 \text { anal. })\end{array}$ \\
\hline$c_{\beta}^{e}$ & 0.8010 & 0.8007 & $\begin{array}{c}0.8004 \\
(0.8004 \text { anal. })\end{array}$ & 0.80007 & 0.80007 & 0.80008 & $\begin{array}{c}0.80039 \\
(0.80040 \text { anal. })\end{array}$ \\
\hline
\end{tabular}

Two different interface widths are considered. For case 256-w1-25, $\mathrm{W}=$ 0.792 and $\kappa=5.6818$ give $\sigma_{\text {int }}=0.5$ and interfacial width $l_{\text {int }}=8.33 \Delta \mathrm{x}$. For case $256-\mathrm{w} 2-25, \mathrm{~W}=0.66$ and $\kappa=6.8182$ give $\sigma_{\text {int }}=0.5$ and $l_{\text {int }}=10$ $\Delta \mathrm{x}$. For case 256-g2-25, along with $\Delta \mathrm{x}=0.1$ and $\Delta \mathrm{t}=1, \mathrm{~W}=7.92$ and $\kappa$ $=5.6818$ give $\sigma_{i n t}=0.5$ and $l_{\text {int }}=8.33 \Delta \mathrm{x}$.

Fig. 10 gives the composition, stress, strain and elastic energy plots obtained from the simulations at equilibrium. The last four columns of Table 4 give the bulk properties for the three cases along with the analytical solution.

The compositions at the interface match well with the analytical solution given by (21) and (22) for all cases. Fig. 10(a) shows the composition profile for Case 256-w1-25. The composition of the precipitate is constant since all 
the elastic properties are also constant inside the precipitate. The composition of the matrix, however, is not constant and shows a slight variation (Fig. 10(b) shows a close-up view), following the trend of the elastic energy profiles that peak at the interface. The composition of the matrix at the interface is calculated as the value at the first point starting from the diffuse interface region as we move away from the centre of the ellipse where the variation is less than 1e-5. Darvishi Kamachali et al. [23] have found that such a profile can be stabilised by mechanically driven fluxes when the stiffness of the matrix varies with composition. In the model used in our work, there is no explicit coupling of the composition with the elastic energy since none of the elastic properties depend directly on composition. The coupling comes about only through the phase-field variable $\phi$. Here too, a similar profile is expected to be stable since the elastic energy varies continuously inside the matrix at equilibrium.

Figs. 10(c), 10(d), and 10(e) give the stress, strain, and elastic energy density profiles respectively, which show better agreement with the analytical solution compared to the first set of simulations with smaller system size. The profiles overlap for different interface widths, thereby showing no significant change. $\sigma_{12}$ distribution for Case 256-w1-25 is given in Fig. 10(f). $\sigma_{12}$ is constant $(=0)$ inside the precipitate as expected, as seen in Fig. 10(f). It peaks at the intersection of the diagonals of the ellipse with the interface, falling off as we move away from the precipitate. This is also as expected from the analytical model.

Most of the bulk properties (Table 4) are now closer to the analytical values due to the larger system size. There is, of course, still a difference since the system is still not large enough to satisfy the infinite matrix assumption. The first number in brackets for the results from simulations indicate the percentage difference between the simulated and analytical values. The second number for Cases 256-w2-25 and 256-g2-25 indicate the percentage difference with respect to Case 256-w1-25. Again, with changes in interface width, there is only less than $1 \%$ difference in the values of most of the bulk properties. The differences in the stress and strain in the bulk do not affect the total elastic energy density of the system, which agrees very well with the analytical solution. This shows that due to the larger interface width, no excess energy is generated from the elastic contribution. 


\section{Conclusions and perpectives}

We formulated and validated a quantitative scheme to treat elastic effects in phase-field models. In quantitative phase-field models, the bulk properties resulting from the simulations do not depend on the value of the diffuse interface width that is chosen and the total energy is formulated such that there is no extra interfacial energy apart from what is expected from the interfacial energy formulation explicitly. By using interfacial conditions consistent with local equilibrium conditions calculated analytically, the new scheme proposed in this work prevents the generation of extra interfacial energy from the elastic energy formulation. This was validated by simulating the following two-phase systems: $2 \mathrm{D}$ and $3 \mathrm{D}$ inhomogeneous systems in the presence of shear and applied strains with a planar interface and 2D systems containing an elliptical second-phase precipitate (non-planar interface). The accuracy of the bulk properties in the two phases did not change significantly with changes in interface width, even though they depended on the system size and the grid refinement. This was tested and proven by performing simulations at different interface widths, different system sizes and different length scales. The results showed that no interface-width-dependent excess interfacial energy was generated due to the elastic energy formulation. This would then allow us to account for physical excess interface stresses independent from those caused by the model formulation. The quantitative scheme presented in this paper can now be applied to any 2D and 3D system irrespective of the nature of elastic moduli, interface curvature (morphology) and the presence of shear strains. This model is currently being combined with plasticity models $[24,25]$ and a multi-phase field model [26] in order to study more complex multi-phase systems with realistic stress states.

\section{Acknowledgements}

The authors thank OT/07/040 and CREA/12/012 for financial support. Simulations were performed using the VSC - Flemish Supercomputer Center, funded by the Hercules foundation and the Flemish Government - department EWI. This work was carried out within the framework of COST MP0602 Advanced Solder Materials for High Temperature Application. A.D. thanks Oleg Shchyglo, Yann Le Bouar, and Benoît Appolaire for fruitful discussions. 


\section{References}

[1] Durga, A., Wollants, P., Moelans, N.. Evaluation of interfacial excess contributions in different phase-field models for elastically inhomogeneous systems. Modelling Simul Mater Sci Eng 2013;21:055018.

[2] Mamivand, M., Zaeem, M.A., Kadiri, H.E.. A review on phase field modeling of martensitic phase transformation. Comp Mater Sci 2013;77:304.

[3] Kim, D.U., Kim, S.G., Kim, W.T., Cho, J., Han, H.N., Cha, P.R.. Effect of micro-elasticity on grain grwoth and texture evolution: a phase field study. Mater Sci Forum 2010;654-656:1590.

[4] Steinbach, I., Apel, M.. Multi phase field model for solid state transformation with elastic strain. Physica D 2006;217:153.

[5] Ammar, K., Appolaire, B., Cailletaud, G., Forest, S.. Combining phase field approach and homogenization methods for modelling phase transformation in elastoplastic media. Eur J Comput Mech 2009;18(56):485.

[6] Hill, R.. Elastic properties of reinforced solids: Some theoretical principles. J Mech Phys Solids 1963;11:357.

[7] Khachaturyan, A.G.. Theory of structural transformations in solids. New York: Wiley; 1983.

[8] Yeon, D.H., Cha, P.R., Kim, J.H., Grant, M., Yoon, J.K.. A phase field model for phase transformation in an elastically stressed binary alloy. Modelling Simul Mater Sci Eng 2005;13:299-319.

[9] Karma, A., Rappel, W.J.. Phase-field method for computationally efficient modeling of solidification with arbitrary interface kinetics. Phys Rev E 1996;53:3017.

[10] Eiken, J., Böttger, B., Steinbach, I.. Multiphase-field approach for multicomponent alloys with extrapolation scheme for numerical application. Phys Rev E 2006;73:066122.

[11] Kim, S.G., Kim, W.T., Suzuki, T.. Phase-field model for binary alloys. Phys Rev E 1999;60:7186. 
[12] Moelans, N., Blanpain, B., Wollants, P.. Quantitative analysis of grain boundary properties in a generalized phase field model for grain growth in anisotropic systems. Phys Rev B 2008;78(2):024113.

[13] Nicoli, M., Plapp, M., Henry, H.. Tensorial mobilities for accurate solution of transport problems in models with diffuse interfaces. Phys Rev E 2011;84:046707.

[14] Johnson, W.C.. Precipitate shape evolution under applied stress thermodynamics and kinetics. Metall Trans A 1987;18A:233.

[15] Jin, X., Wang, Z., Zhou, Q., Keer, L.M., Wang, Q.. On the solution of an elliptical inhomogeneity in plane elasticity by the equivalent inclusion method. J Elasticity 2014;114(1):1.

[16] Cahn, J.W., Larché, F.. A simple model for coherent equilibrium. Acta Metall 1984;32(11):1915.

[17] Jaswon, M.A., Bhargava, R.D.. Two-dimensional elastic inclusion problems. Math Proc Cambridge 1961;57:669.

[18] Eshelby, J.D.. The determination of the elastic field of an ellipsoidal inclusion, and related problems. Proc Roy Soc A 1957;241:376.

[19] J. Mosler and O. Shchyglo and H. Montazer Hojjat, . A novel homogenization method for phase field approaches based on partial rank-one relaxation. J Mech Phys Solids 2014;68:251.

[20] Brits, L.. Euler angles. http://en.wikipedia.org/wiki/File:Eulerangles.svg; 2008. @ .

[21] Hu, S.Y., Chen, L.Q.. A phase-field model for evolving microstructures with strong elastic inhomogeneity. Acta Mater 2001;49:1879.

[22] Gururajan, M.P.. Elastic inhomogeneity effects on microstructures a phase field study. Ph.D. thesis; Department of Metallurgy, Indian Institute of Science; Bangalore - 560 012, India; 2006.

[23] R. Darvishi Kamachali and E. Borukhovich and O. Shchyglo and I. Steinbach, . Solutal gradients in strained equilibrium. Phil Mag Lett 2013;93(12):680. 
[24] Yeddu, H.K., Malik, A., Ȧgren, J., Amberg, G., Borgenstam, A.. Three-dimensional phase-field modeling of martensitic microstructure evolution in steels. Acta Mater 2012;60:1538.

[25] Gaubert, A., Bouar, Y.L., Finel, A.. Coupling phase field and viscoplasticity to study rafting in ni-based superalloys. Phil Mag 2010;90(1-4):375.

[26] Moelans, N.. A quantitative and thermodynamically consistent phase-field interpolation function for multi-phase systems. Acta Mater 2011;59:1077.

[27] P. Van Houtte, . Continuum modelling of material properties. 2010. Course notes of Spring 2010 semester at KULeuven. 


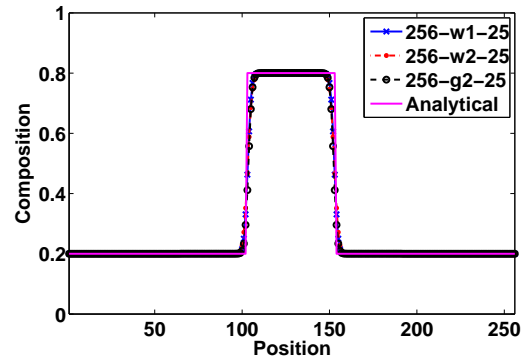

(a)

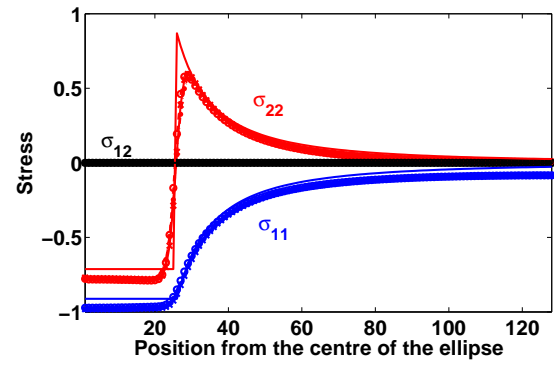

(c)

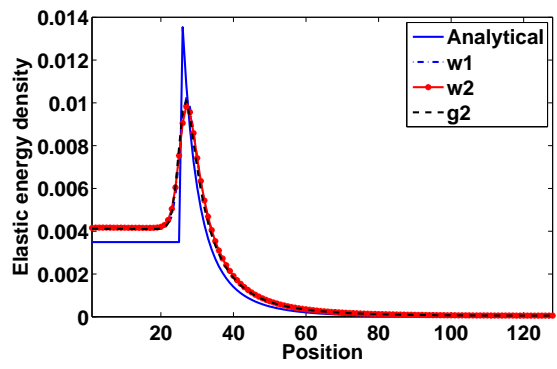

(e)

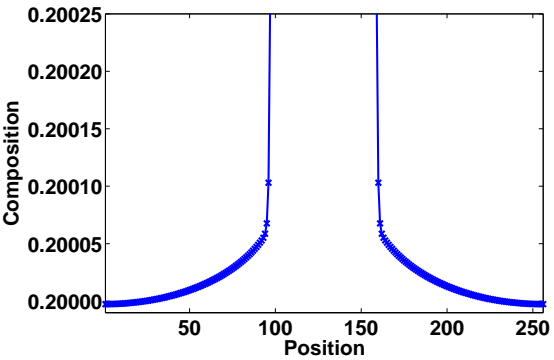

(b)

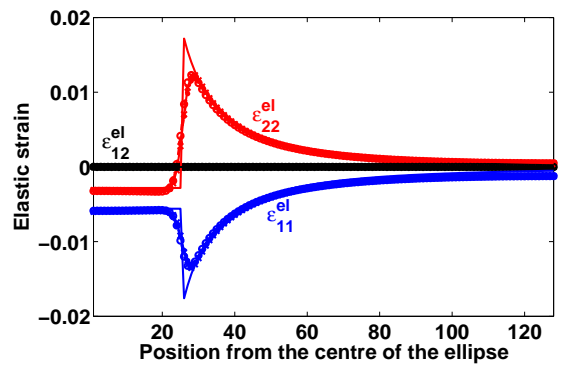

(d)

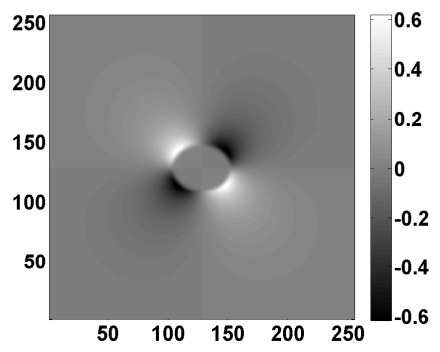

(f)

Figure 10: Simulation results obtained for a 2D system with an elliptical precipitate with a $=25$ and $\mathrm{b}=20$ in a matrix of size $256 \times 256$ grid points for different interface widths: (a) Composition follows the analytical solution (analytical profile shown only for the length scale corresponding to cases 256-w1-25 and 256-w2-25) closely at the interface for all interface widths but (b) a close-up view (shown here for 256-w1-25) shows that it is not constant in the matrix due to elastic energy variation. (c) Stress, (d) elastic strain, and (e) elastic energy profiles overlap for different interface widths (same markers are used to indicate the profiles for the different cases as those in the composition profiles). There is a deviation from the analytical solution for all cases due to the limited resolution but changing the interface width does not change the bulk properties significantly. (f) $\sigma_{12}$ (shown for Case 256-w1-25) is zero inside the precipitate and peaks at the interface at the diagonals of the ellipse as expected from the analytical model. 


\section{Appendix A. Solving stresses and strains for the new scheme in $3 \mathrm{D}$}

Using the matrix notation [27], we can reduce the number of indices used for representing the stiffness, strain and stress. The following substitutions are made: ' 11 ' $\rightarrow$ ' 1 ', '22' $\rightarrow$ '2', ' 33 ' $\rightarrow$ ' 3 ', ' 23 ' $\rightarrow$ ' 4 ', ' 13 ' $\rightarrow{ }^{~ ' 5 ' ~ a n d ~ ' 12 ' ~}$ $\rightarrow$ ' 6 '. For instance, $C_{1112}$ is written as $C_{16}$. For the stiffness, the matrix notation $C_{p q}=C_{i j k l}$, which is the tensor notation, for all p and q. For the stresses, $\sigma_{p}=\sigma_{i j}$ for all p. For the strains, $\epsilon_{p}^{e l}=\epsilon_{i j}^{e l}$ for $\mathrm{p} \leq 3$ and $\epsilon_{p}^{e l}=2 \epsilon_{i j}$ for $\mathrm{p}>3$. For instance, $2 \epsilon_{13}^{e l}$ in the tensor notation is written as $\epsilon_{5}^{e l}$ in the matrix notation. Finally, the compliance $S_{p q}=S_{i j k l}$ if both p and q $\leq 3, S_{p q}$ $=2 S_{i j k l}$ if only p or q $\leq 3$ and $S_{p q}=4 S_{i j k l}$ if both p and q $>3$. Also, $S_{i j k l}$ $=S_{i j l k}=S_{j i k l}=S_{k l i j}$ and $C_{i j k l}=C_{i j l k}=C_{j i k l}=C_{k l i j}$ due to symmetry.

Rewriting (40) - (45), which relate the unknown stresses and strains in the new scheme with the known values, in matrix notation (superscript $\rho$ denotes $\alpha$ or $\beta$ ),

$$
\begin{aligned}
\sigma_{2}^{\rho}= & C_{21}^{\rho} \epsilon_{1}^{e l, \rho}+C_{22}^{\rho} \epsilon_{2}^{e l, V T S, \rho}+C_{23}^{\rho} \epsilon_{3}^{e l, V T S, \rho} \\
& +C_{24}^{\rho} \epsilon_{4}^{e l, V T S, \rho}+C_{25}^{\rho} \epsilon_{5}^{e l, \rho}+C_{26}^{\rho} \epsilon_{6}^{e l, \rho}, \\
\sigma_{3}^{\rho}= & C_{31}^{\rho} \epsilon_{1}^{e l, \rho}+C_{32}^{\rho} \epsilon_{2}^{e l, V T S, \rho}+C_{33}^{\rho} \epsilon_{3}^{e l, V T S, \rho} \\
& +C_{34}^{\rho} \epsilon_{4}^{e l, V T S, \rho}+C_{35}^{\rho} \epsilon_{5}^{e l, \rho}+C_{36}^{\rho} \epsilon_{6}^{e l, \rho}, \\
\sigma_{4}^{\rho}= & C_{41}^{\rho} \epsilon_{1}^{e l, \rho}+C_{42}^{\rho} \epsilon_{2}^{e l, V T S, \rho}+C_{43}^{\rho} \epsilon_{3}^{e l, V T S, \rho} \\
& +C_{44}^{\rho} \epsilon_{4}^{e l, V T S, \rho}+C_{45}^{\rho} \epsilon_{5}^{e l, \rho}+C_{46}^{\rho} \epsilon_{6}^{e l, \rho}, \\
\epsilon_{1}^{e l, \rho}= & S_{11}^{\rho} \sigma_{1}^{S A S}+S_{12}^{\rho} \sigma_{2}^{\rho}+S_{13}^{\rho} \sigma_{3}^{\rho}+S_{14}^{\rho} \sigma_{4}^{\rho}+S_{15}^{\rho} \sigma_{5}^{S A S}+S_{16}^{\rho} \sigma_{6}^{S A S}, \\
\epsilon_{5}^{e l, \rho}= & S_{51}^{\rho} \sigma_{1}^{S A S}+S_{52}^{\rho} \sigma_{2}^{\rho}+S_{53}^{\rho} \sigma_{3}^{\rho}+S_{54}^{\rho} \sigma_{4}^{\rho}+S_{55}^{\rho} \sigma_{5}^{S A S}+S_{56}^{\rho} \sigma_{6}^{S A S}, \\
\epsilon_{6}^{e l, \rho}= & S_{61}^{\rho} \sigma_{1}^{S A S}+S_{62}^{\rho} \sigma_{2}^{\rho}+S_{63}^{\rho} \sigma_{3}^{\rho}+S_{64}^{\rho} \sigma_{4}^{\rho}+S_{65}^{\rho} \sigma_{5}^{S A S}+S_{66}^{\rho} \sigma_{6}^{S A S}
\end{aligned}
$$

Substituting for the stresses in the strain expressions and rewriting, we obtain

$$
\begin{aligned}
& L_{11}^{\rho} \epsilon_{1}^{e l, \rho}+L_{12}^{\rho} \epsilon_{5}^{e l, \rho}+L_{13}^{\rho} \epsilon_{6}^{e l, \rho}=R_{1}^{\rho}, \\
& L_{21}^{\rho} \epsilon_{1}^{e l, \rho}+L_{22}^{\rho} \epsilon_{5}^{e l, \rho}+L_{23}^{\rho} \epsilon_{6}^{e l, \rho}=R_{2}^{\rho}, \\
& L_{31}^{\rho} \epsilon_{1}^{e l, \rho}+L_{32}^{\rho} \epsilon_{5}^{e l, \rho}+L_{33}^{\rho} \epsilon_{6}^{e l, \rho}=R_{3}^{\rho},
\end{aligned}
$$


where

$$
\begin{aligned}
& L_{11}^{\rho}=1-S_{12}^{\rho} C_{21}^{\rho}-S_{13}^{\rho} C_{31}^{\rho}-S_{14}^{\rho} C_{41}^{\rho}, \\
& L_{12}^{\rho}=-S_{12}^{\rho} C_{25}^{\rho}-S_{13}^{\rho} C_{35}^{\rho}-S_{14}^{\rho} C_{45}^{\rho} \text {, } \\
& L_{13}^{\rho}=-S_{12}^{\rho} C_{26}^{\rho}-S_{13}^{\rho} C_{36}^{\rho}-S_{14}^{\rho} C_{46}^{\rho} \text {, } \\
& L_{21}^{\rho}=-S_{52}^{\rho} C_{21}^{\rho}-S_{53}^{\rho} C_{31}^{\rho}-S_{54}^{\rho} C_{41}^{\rho} \text {, } \\
& L_{22}^{\rho}=1-S_{52}^{\rho} C_{25}^{\rho}-S_{53}^{\rho} C_{35}^{\rho}-S_{54}^{\rho} C_{45}^{\rho} \text {, } \\
& L_{23}^{\rho}=-S_{52}^{\rho} C_{26}^{\rho}-S_{53}^{\rho} C_{36}^{\rho}-S_{54}^{\rho} C_{46}^{\rho} \text {, } \\
& L_{31}^{\rho}=-S_{62}^{\rho} C_{21}^{\rho}-S_{63}^{\rho} C_{31}^{\rho}-S_{64}^{\rho} C_{41}^{\rho} \text {, } \\
& L_{32}^{\rho}=-S_{62}^{\rho} C_{25}^{\rho}-S_{63}^{\rho} C_{35}^{\rho}-S_{64}^{\rho} C_{45}^{\rho} \text {, } \\
& L_{33}^{\rho}=1-S_{62}^{\rho} C_{26}^{\rho}-S_{63}^{\rho} C_{36}^{\rho}-S_{64}^{\rho} C_{46}^{\rho} \text {, } \\
& R_{1}^{\rho}=S_{11}^{\rho} \sigma_{1}^{S A S}+S_{15}^{\rho} \sigma_{5}^{S A S}+S_{16}^{\rho} \sigma_{6}^{S A S}+S_{12}^{\rho}\left(C_{22}^{\rho} \epsilon_{2}^{e l, V T S, \rho}+C_{23}^{\rho} \epsilon_{3}^{e l, V T S, \rho}+C_{24}^{\rho} \epsilon_{4}^{e l, V T S, \rho}\right) \\
& +S_{13}^{\rho}\left(C_{32}^{\rho} \epsilon_{2}^{e l, V T S, \rho}+C_{33}^{\rho} \epsilon_{3}^{e l, V T S, \rho}+C_{34}^{\rho} \epsilon_{4}^{e l, V T S, \rho}\right) \\
& +S_{14}^{\rho}\left(C_{42}^{\rho} \epsilon_{2}^{e l, V T S, \rho}+C_{43}^{\rho} \epsilon_{3}^{e l, V T S, \rho}+C_{44}^{\rho} \epsilon_{4}^{e l, V T S, \rho}\right), \\
& R_{2}^{\rho}=S_{51}^{\rho} \sigma_{1}^{S A S}+S_{55}^{\rho} \sigma_{5}^{S A S}+S_{56}^{\rho} \sigma_{6}^{S A S}+S_{52}^{\rho}\left(C_{22}^{\rho} \epsilon_{2}^{e l, V T S, \rho}+C_{23}^{\rho} \epsilon_{3}^{e l, V T S, \rho}+C_{24}^{\rho} \epsilon_{4}^{e l, V T S, \rho}\right) \\
& +S_{53}^{\rho}\left(C_{32}^{\rho} \epsilon_{2}^{e l, V T S, \rho}+C_{33}^{\rho} \epsilon_{3}^{e l, V T S, \rho}+C_{34}^{\rho} \epsilon_{4}^{e l, V T S, \rho}\right) \\
& +S_{54}^{\rho}\left(C_{42}^{\rho} \epsilon_{2}^{e l, V T S, \rho}+C_{43}^{\rho} \epsilon_{3}^{e l, V T S, \rho}+C_{44}^{\rho} \epsilon_{4}^{e l, V T S, \rho}\right), \\
& R_{3}^{\rho}=S_{61}^{\rho} \sigma_{1}^{S A S}+S_{65}^{\rho} \sigma_{5}^{S A S}+S_{66}^{\rho} \sigma_{6}^{S A S}+S_{62}^{\rho}\left(C_{22}^{\rho} \epsilon_{2}^{e l, V T S, \rho}+C_{23}^{\rho} \epsilon_{3}^{e l, V T S, \rho}+C_{24}^{\rho} \epsilon_{4}^{e l, V T S, \rho}\right) \\
& +S_{63}^{\rho}\left(C_{32}^{\rho} \epsilon_{2}^{e l, V T S, \rho}+C_{33}^{\rho} \epsilon_{3}^{e l, V T S, \rho}+C_{34}^{\rho} \epsilon_{4}^{e l, V T S, \rho}\right) \\
& +S_{64}^{\rho}\left(C_{42}^{\rho} \epsilon_{2}^{e l, V T S, \rho}+C_{43}^{\rho} \epsilon_{3}^{e l, V T S, \rho}+C_{44}^{\rho} \epsilon_{4}^{e l, V T S, \rho}\right) .
\end{aligned}
$$

The strains are given as:

$$
\left(\begin{array}{c}
\epsilon_{1}^{e l, \rho} \\
\epsilon_{5}^{e l, \rho} \\
\epsilon_{6}^{l l, \rho}
\end{array}\right)=\left(\begin{array}{lll}
L_{11}^{\rho} & L_{12}^{\rho} & L_{13}^{\rho} \\
L_{21}^{\rho} & L_{22}^{\rho} & L_{23}^{\rho} \\
L_{31}^{\rho} & L_{32}^{\rho} & L_{33}^{\rho}
\end{array}\right)^{-1}\left(\begin{array}{c}
R_{1}^{\rho} \\
R_{2}^{\rho} \\
R_{3}^{\rho}
\end{array}\right) .
$$

The stresses can then be calculated using (A.1) - (A.3). 


\section{Appendix B. Calculation of strain and stress derivatives in 3D}

The derivatives of $\sigma_{p}^{S A S}$ are given as:

$$
\begin{aligned}
\frac{\partial \sigma_{1}^{S A S}}{\partial \phi}= & C_{11}^{\alpha} \frac{\partial \epsilon_{1}^{e l, S A S, \alpha}}{\partial \phi}+C_{12}^{\alpha} \frac{\partial \epsilon_{2}^{e l, S A S, \alpha}}{\partial \phi}+C_{13}^{\alpha} \frac{\partial \epsilon_{3}^{e l, S A S, \alpha}}{\partial \phi} \\
& +C_{14}^{\alpha} \frac{\partial \epsilon_{4}^{e l, S A S, \alpha}}{\partial \phi}+C_{15}^{\alpha} \frac{\partial \epsilon_{5}^{e l, S A S, \alpha}}{\partial \phi}+C_{16}^{\alpha} \frac{\partial \epsilon_{6}^{e l, S A S, \alpha}}{\partial \phi}, \\
\frac{\partial \sigma_{5}^{S A S}}{\partial \phi}= & C_{51}^{\alpha} \frac{\partial \epsilon_{1}^{e l, S A S, \alpha}}{\partial \phi}+C_{52}^{\alpha} \frac{\partial \epsilon_{2}^{e l, S A S, \alpha}}{\partial \phi}+C_{53}^{\alpha} \frac{\partial \epsilon_{3}^{e l, S A S, \alpha}}{\partial \phi} \\
& +C_{54}^{\alpha} \frac{\partial \epsilon_{4}^{e l, S A S, \alpha}}{\partial \phi}+C_{55}^{\alpha} \frac{\partial \epsilon_{5}^{e l, S A S, \alpha}}{\partial \phi}+C_{56}^{\alpha} \frac{\partial \epsilon_{6}^{e l, S A S, \alpha}}{\partial \phi}, \\
\frac{\partial \sigma_{6}^{S A S}}{\partial \phi}= & C_{61}^{\alpha} \frac{\partial \epsilon_{1}^{e l, S A S, \alpha}}{\partial \phi}+C_{62}^{\alpha} \frac{\partial \epsilon_{2}^{e l, S A S, \alpha}}{\partial \phi}+C_{63}^{\alpha} \frac{\partial \epsilon_{3}^{e l, S A S, \alpha}}{\partial \phi} \\
& +C_{64}^{\alpha} \frac{\partial \epsilon_{4}^{e l, S A S, \alpha}}{\partial \phi}+C_{65}^{\alpha} \frac{\partial \epsilon_{5}^{e l, S A S, \alpha}}{\partial \phi}+C_{66}^{\alpha} \frac{\partial \epsilon_{6}^{e l, S A S, \alpha}}{\partial \phi},
\end{aligned}
$$

where

$$
\begin{aligned}
\frac{\partial \epsilon_{p}^{e l, S A S, \alpha}}{\partial \phi}= & {\left[p(\phi) I+(1-p(\phi)) M_{p q}\right]^{-1} } \\
& {\left[-p(\phi)\left(\epsilon_{q}^{*, \alpha}-\epsilon_{q}^{*, \beta}\right)-p^{\prime}(\phi)\left(\epsilon_{q}^{e l, S A S, \alpha}-\epsilon_{q}^{e l, S A S, \beta}\right)\right], } \\
M_{p q}= & {\left[C_{p k}^{\beta}\right]^{-1} C_{k q}^{\alpha} . }
\end{aligned}
$$

The derivatives of $\epsilon_{1}^{e l}, \epsilon_{5}^{e l}$ and $\epsilon_{6}^{e l}$ are given in terms of $\frac{\partial \sigma_{p}^{S A S}}{\partial \phi}$ as:

$$
\left(\begin{array}{c}
\frac{\partial \epsilon_{1}^{e l, \rho}}{\partial \phi} \\
\frac{\partial \epsilon_{5}^{e l, \rho}}{\partial \phi} \\
\frac{\partial \epsilon_{6}^{e l, \rho}}{\partial \phi}
\end{array}\right)=\left(\begin{array}{lll}
L_{11}^{\rho} & L_{12}^{\rho} & L_{13}^{\rho} \\
L_{21}^{\rho} & L_{22}^{\rho} & L_{23}^{\rho} \\
L_{31}^{\rho} & L_{32}^{\rho} & L_{33}^{\rho}
\end{array}\right)^{-1}\left(\begin{array}{c}
\frac{\partial R_{1}^{\rho}}{\partial \phi} \\
\frac{\partial R_{2}^{\rho}}{\partial \phi} \\
\frac{\partial R_{3}^{\rho}}{\partial \phi}
\end{array}\right),
$$

where

$$
\begin{aligned}
\frac{\partial R_{1}^{\rho}}{\partial \phi} & =S_{11}^{\rho} \frac{\partial \sigma_{1}^{S A S}}{\partial \phi}+S_{15}^{\rho} \frac{\partial \sigma_{5}^{S A S}}{\partial \phi}+S_{16}^{\rho} \frac{\partial \sigma_{6}^{S A S}}{\partial \phi}, \\
\frac{\partial R_{2}^{\rho}}{\partial \phi} & =S_{51}^{\rho} \frac{\partial \sigma_{1}^{S A S}}{\partial \phi}+S_{55}^{\rho} \frac{\partial \sigma_{5}^{S A S}}{\partial \phi}+S_{56}^{\rho} \frac{\partial \sigma_{6}^{S A S}}{\partial \phi}, \\
\frac{\partial R_{3}^{\rho}}{\partial \phi} & =S_{61}^{\rho} \frac{\partial \sigma_{1}^{S A S}}{\partial \phi}+S_{65}^{\rho} \frac{\partial \sigma_{5}^{S A S}}{\partial \phi}+S_{66}^{\rho} \frac{\partial \sigma_{6}^{S A S}}{\partial \phi} .
\end{aligned}
$$


Finally, the stress derivatives $\frac{\partial \sigma_{2}^{\rho}}{\partial \phi}, \frac{\partial \sigma_{3}^{\rho}}{\partial \phi}$ and $\frac{\partial \sigma_{4}^{\rho}}{\partial \phi}$ are given as:

$$
\begin{aligned}
\frac{\partial \sigma_{2}^{\rho}}{\partial \phi} & =C_{21}^{\rho} \frac{\partial \epsilon_{1}^{e l, \rho}}{\partial \phi}+C_{25}^{\rho} \frac{\partial \epsilon_{5}^{e l, \rho}}{\partial \phi}+C_{26}^{\rho} \frac{\partial \epsilon_{6}^{e l, \rho}}{\partial \phi} \\
\frac{\partial \sigma_{3}^{\rho}}{\partial \phi} & =C_{31}^{\rho} \frac{\partial \epsilon_{1}^{e l, \rho}}{\partial \phi}+C_{35}^{\rho} \frac{\partial \epsilon_{5}^{e l, \rho}}{\partial \phi}+C_{36}^{\rho} \frac{\partial \epsilon_{6}^{e l, \rho}}{\partial \phi} \\
\frac{\partial \sigma_{4}^{\rho}}{\partial \phi} & =C_{41}^{\rho} \frac{\partial \epsilon_{1}^{e l, \rho}}{\partial \phi}+C_{45}^{\rho} \frac{\partial \epsilon_{5}^{e l, \rho}}{\partial \phi}+C_{46}^{\rho} \frac{\partial \epsilon_{6}^{e l, \rho}}{\partial \phi} .
\end{aligned}
$$

\section{Appendix C. New scheme formulation in 2D}

According to Johnson's model in 2D, only the stress components $\sigma_{11}$ and $\sigma_{12}$ are equal in the two phases. Therefore, in the new scheme, $\sigma_{11}$ and $\sigma_{12}$ are calculated using SAS $\left(\sigma_{11}^{\alpha}=\sigma_{11}^{\beta}=\sigma_{11}^{S A S}, \sigma_{12}^{\alpha}=\sigma_{12}^{\beta}=\sigma_{12}^{S A S}\right)$. The total strain component, $\epsilon_{22}=\delta \epsilon_{22}+\overline{\epsilon_{22}}$, is equal in the two phases. Therefore, $\epsilon_{22}$ is calculated using VTS $\left(\epsilon_{22}^{\alpha}=\epsilon_{22}^{\beta}=\epsilon_{22}^{V T S}\right)$. Then, $\epsilon_{22}^{e l, \alpha}=\epsilon_{22}^{V T S}-\epsilon_{22}^{*, \alpha}$ and $\epsilon_{22}^{e l, \beta}=\epsilon_{22}^{V T S}-\epsilon_{22}^{*, \beta}$, denoted as $\epsilon_{22}^{e l, V T S, \alpha}$ and $\epsilon_{22}^{e l, V T S, \beta}$ respectively henceforth.

Due to these additional relations due to the shear stresses and strains when compared to the first version of the model proposed in [1], $\sigma_{22}, \epsilon_{11}^{e l}$ and $\epsilon_{12}^{e l}$ for the $\alpha$ phase are reformulated as follows:

$$
\begin{aligned}
\sigma_{22}^{\alpha} & =C_{1122}^{\alpha} \epsilon_{11}^{e l, \alpha}+C_{2222}^{\alpha} \epsilon_{22}^{V T S, \alpha}+2 C_{2212}^{\alpha} \epsilon_{12}^{e l, \alpha}, \\
\epsilon_{11}^{e l, \alpha} & =S_{1111}^{\alpha} \sigma_{11}^{S A S}+S_{1122}^{\alpha} \sigma_{22}^{\alpha}+2 S_{1112}^{\alpha} \sigma_{12}^{S A S}, \\
\epsilon_{12}^{e l, \alpha} & =S_{1112}^{\alpha} \sigma_{11}^{S A S}+S_{2212}^{\alpha} \sigma_{22}^{\alpha}+2 S_{1212}^{\alpha} \sigma_{12}^{S A S} .
\end{aligned}
$$

$\sigma_{22}^{\alpha}$ can be calculated in terms of the known quantities $\sigma_{11}^{S A S}, \sigma_{12}^{S A S}$ and
$\epsilon_{22}^{e l, V T S, \alpha}$ as

$$
\begin{aligned}
\sigma_{22}^{\alpha}= & \frac{C_{1122}^{\alpha}\left(S_{1111}^{\alpha} \sigma_{11}^{S A S}+2 S_{1112}^{\alpha} \sigma_{12}^{S A S}\right)+C_{2222}^{\alpha} \epsilon_{22}^{e l, V T S, \alpha}}{1-C_{1122}^{\alpha} S_{1122}^{\alpha}-2 C_{2212}^{\alpha} S_{2212}^{\alpha}} \\
& +\frac{2 C_{2212}^{\alpha}\left(S_{1112}^{\alpha} \sigma_{11}^{S A S}+2 S_{1212}^{\alpha} \sigma_{12}^{S A S}\right)}{1-C_{1122}^{\alpha} S_{1122}^{\alpha}-2 C_{2212}^{\alpha} S_{2212}^{\alpha}}
\end{aligned}
$$

$\epsilon_{11}^{e l, \alpha}$ and $\epsilon_{12}^{e l, \alpha}$ can be computed using (C.2) - (C.3). $\sigma_{22}^{\beta}, \epsilon_{11}^{e l, \beta}$ and $\epsilon_{12}^{\beta}$ are also calculated using appropriate relations for the $\beta$ phase. 
The elastic energy density is interpolated as:

$$
\begin{aligned}
f^{e l}= & p(\phi) f_{\alpha}^{e l}+[1-p(\phi)] f_{\beta}^{e l} \\
= & p(\phi) \frac{1}{2}\left(\sigma_{11}^{S A S, \alpha} \epsilon_{11}^{e l, \alpha}+\sigma_{22}^{\alpha} \epsilon_{22}^{e l, V T S, \alpha}+2 \sigma_{12}^{S A S, \alpha} \epsilon_{12}^{e l, \alpha}\right) \\
& +[1-p(\phi)] \frac{1}{2}\left(\sigma_{11}^{S A S, \beta} \epsilon_{11}^{e l, \beta}+\sigma_{22}^{\beta} \epsilon_{22}^{e l, V T S, \beta}+2 \sigma_{12}^{S A S, \beta} \epsilon_{12}^{e l, \beta}\right),
\end{aligned}
$$

and its derivative with respect to $\phi$ is accordingly:

$$
\begin{aligned}
\frac{\partial f^{e l}}{\partial \phi}= & p^{\prime}(\phi)\left(f_{\alpha}^{e l}-f_{\beta}^{e l}\right) \\
& +\frac{1}{2} p(\phi)\left[\frac{\partial \sigma_{11}^{S A S, \alpha}}{\partial \phi} \epsilon_{11}^{e l, \alpha}+\sigma_{11}^{S A S, \alpha} \frac{\partial \epsilon_{11}^{e l, \alpha}}{\partial \phi}+\frac{\partial \sigma_{22}^{\alpha}}{\partial \phi} \epsilon_{22}^{e l, V T S, \alpha}\right] \\
& +\frac{1}{2} p(\phi)\left[2 \frac{\partial \sigma_{12}^{S A S, \alpha}}{\partial \phi} \epsilon_{12}^{e l, \alpha}+2 \sigma_{12}^{S A S, \alpha} \frac{\partial \epsilon_{12}^{e l, \alpha}}{\partial \phi}\right] \\
& +\frac{1}{2}[1-p(\phi)]\left[\frac{\partial \sigma_{11}^{S A S, \beta}}{\partial \phi} \epsilon_{11}^{e l, \beta}+\sigma_{11}^{S A S, \beta} \frac{\partial \epsilon_{11}^{e l, \beta}}{\partial \phi}+\frac{\partial \sigma_{22}^{\beta}}{\partial \phi} \epsilon_{22}^{e l, V T S, \beta}\right] \\
& +\frac{1}{2}[1-p(\phi)]\left[2 \frac{\partial \sigma_{12}^{S A S, \beta}}{\partial \phi} \epsilon_{12}^{e l, \beta}+2 \sigma_{12}^{S A S, \beta} \frac{\partial \epsilon_{12}^{e l, \beta}}{\partial \phi}\right] .
\end{aligned}
$$


The stress and strain derivatives are given as:

$$
\begin{aligned}
\frac{\partial \sigma_{22}^{\alpha}}{\partial \phi}= & \frac{C_{1122}^{\alpha}\left(S_{1111}^{\alpha} \frac{\partial \sigma_{11}^{S A S}}{\partial \phi}+2 S_{1112}^{\alpha} \frac{\partial \sigma_{12}^{S A S}}{\partial \phi}\right)}{1-C_{1122}^{\alpha} S_{1122}^{\alpha}-2 C_{2212}^{\alpha} S_{2212}^{\alpha}} \\
& +\frac{2 C_{2212}^{\alpha}\left(S_{1112}^{\alpha} \frac{\partial \sigma_{11}^{S A S}}{\partial \phi}+2 S_{1212}^{\alpha} \frac{\partial \sigma_{12}^{S A S}}{\partial \phi}\right)}{1-C_{1122}^{\alpha} S_{1122}^{\alpha}-2 C_{2212}^{\alpha} S_{2212}^{\alpha}}, \\
\frac{\partial \epsilon_{11}^{e l, \alpha}}{\partial \phi}= & S_{1111}^{\alpha} \frac{\partial \sigma_{11}^{S A S}}{\partial \phi}+S_{1122}^{\alpha} \frac{\partial \sigma_{22}^{\alpha}}{\partial \phi}+2 S_{1112}^{\alpha} \frac{\partial \sigma_{12}^{S A S}}{\partial \phi}, \\
\frac{\partial \epsilon_{12}^{e l, \alpha}}{\partial \phi}= & S_{1112}^{\alpha} \frac{\partial \sigma_{11}^{S A S}}{\partial \phi}+S_{2212}^{\alpha} \frac{\partial \sigma_{22}^{\alpha}}{\partial \phi}+2 S_{1212}^{\alpha} \frac{\partial \sigma_{12}^{S A S}}{\partial \phi}, \quad \text { (C.9) } \\
\frac{\partial \sigma_{11}^{S A S, \alpha}}{\partial \phi}= & C_{1111}^{\alpha} \frac{\partial \epsilon_{11}^{e l, S A S, \alpha}}{\partial \phi}+C_{1122}^{\alpha} \frac{\partial \epsilon_{22}^{e l, S A S, \alpha}}{\partial \phi}+2 C_{1112}^{\alpha} \frac{\partial \epsilon_{12}^{e l, S A S, \alpha}}{\partial \phi}(\mathrm{C} .11) \\
\frac{\partial \sigma_{12}^{S A S, \alpha}}{\partial \phi}= & C_{1112}^{\alpha} \frac{\partial \epsilon_{11}^{e l, S A S, \alpha}}{\partial \phi}+C_{2212}^{\alpha} \frac{\partial \epsilon_{22}^{e l, S A S, \alpha}}{\partial \phi}+2 C_{1212}^{\alpha} \frac{\partial \epsilon_{12}^{e l, S A S, \alpha}}{\partial \phi}(\mathrm{C} .12) \\
\text { with } \frac{\partial \epsilon_{k l}^{e l, S A S, \alpha}}{\partial \phi}= & {\left[p(\phi) I+(1-p(\phi)) M_{k l m n}\right]^{-1} } \\
& {\left[-p^{\prime}(\phi)\left(\epsilon_{m n}^{*, \alpha}-\epsilon_{m n}^{*, \beta}\right)-p^{\prime}(\phi)\left(\epsilon_{m n}^{e l, S A S, \alpha}-\epsilon_{m n}^{e l, S A S, \beta}\right)\right](\mathrm{C} .13) }
\end{aligned}
$$

where $M_{k l m n}=\left[C_{i j k l}^{\beta}\right]^{-1} C_{i j m n}^{\alpha}$. 